Provided for non-commercial research and education use. Not for reproduction, distribution or commercial use.

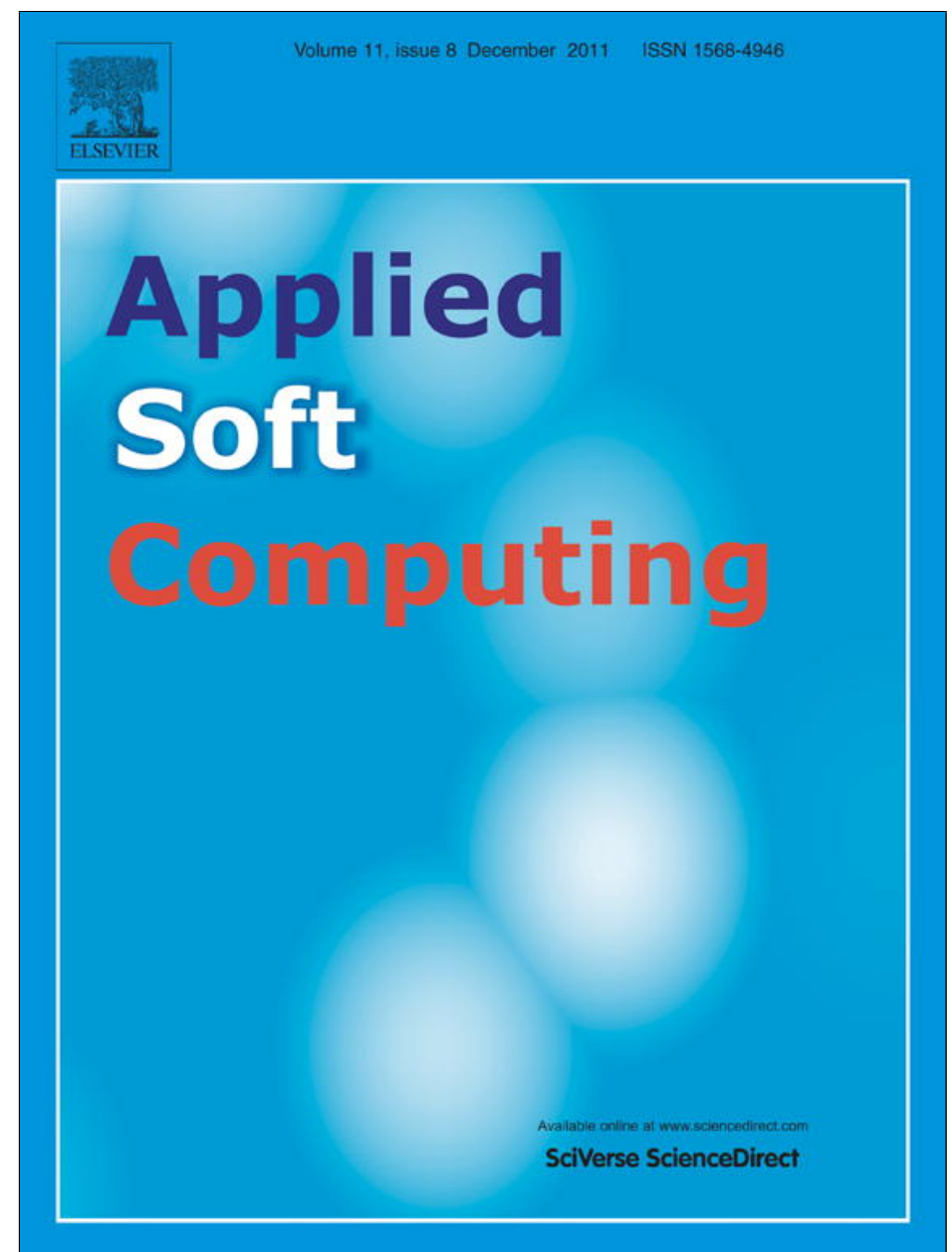

This article appeared in a journal published by Elsevier. The attached copy is furnished to the author for internal non-commercial research and education use, including for instruction at the authors institution and sharing with colleagues.

Other uses, including reproduction and distribution, or selling or licensing copies, or posting to personal, institutional or third party websites are prohibited.

In most cases authors are permitted to post their version of the article (e.g. in Word or Tex form) to their personal website or institutional repository. Authors requiring further information regarding Elsevier's archiving and manuscript policies are encouraged to visit:

http://www.elsevier.com/copyright 
Review article

\title{
Human scalp EEG processing: Various soft computing approaches
}

\author{
Kaushik Majumdar* \\ Indian Statistical Institute, 8th Mile, Mysore Road, Bangalore 560059, India
}

\section{A R T I C L E I N F O}

\section{Article history:}

Received 31 July 2009

Received in revised form 22 April 2011

Accepted 17 July 2011

Available online 30 July 2011

\section{Keywords:}

Bayesian inference

Electroencephalogram (EEG)

Evolutionary computation

Fuzzy clustering

Artificial neural network (ANN)

Neuro-fuzzy techniques

Statistical discrimination

Support vector machine (SVM)

\begin{abstract}
A B S T R A C T
Presently high density EEG systems are available at affordable cost, with which the data dimension has gone up considerably. For efficient computation of this high-dimensional data, various soft computing paradigms are receiving increasing attention. In this survey we have identified certain soft computing techniques (by soft computing techniques we mean computational techniques that take into account the inherent uncertainties in the data and/or in the computing model) for pattern recognition/data mining, such as, neural networks, fuzzy logic, evolutionary computation, statistical discrimination and Bayesian inference, which have turned out to be particularly useful in processing human scalp EEG. Wherever possible results of comparative studies among various techniques have been presented. Analyses of EEG for various feature extraction are exceedingly challenging pattern recognition tasks. This survey has shown that on an average the artificial neural networks and Bayesian approaches have emerged more successful in EEG analysis than the other soft computing paradigms. For readability the paper has been kept as little technical as possible. Large number of references have been listed to aid searching for the technical details.
\end{abstract}

(c) 2011 Elsevier B.V. All rights reserved.

\section{Contents}

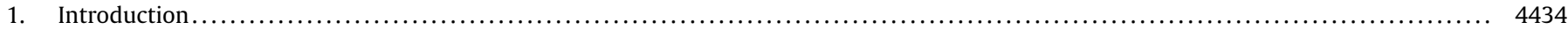

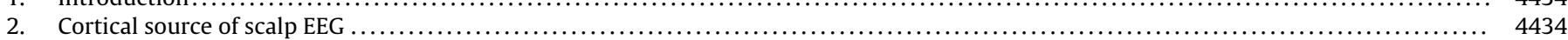

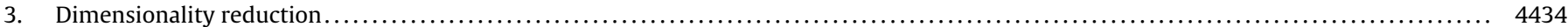

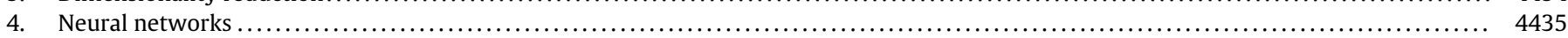

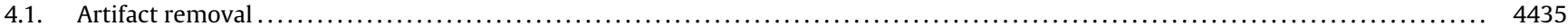

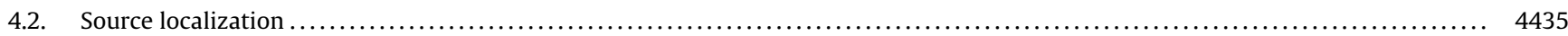

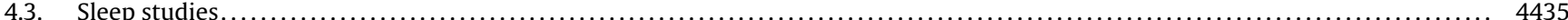

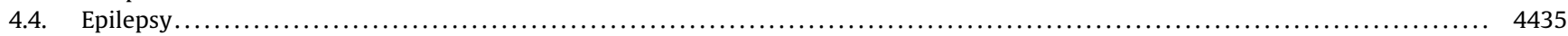

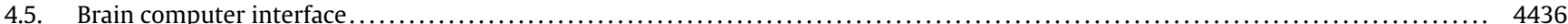

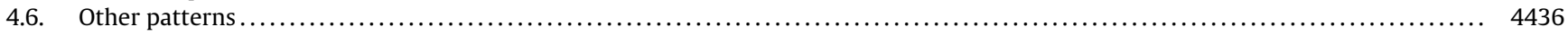

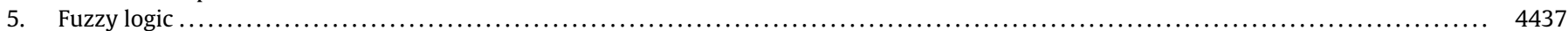

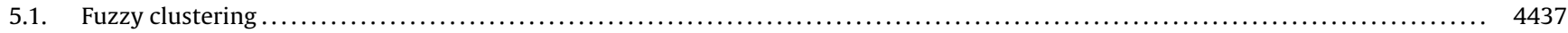

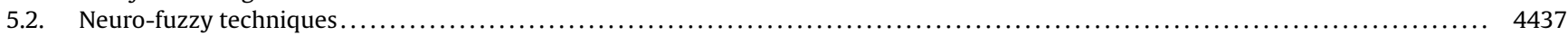

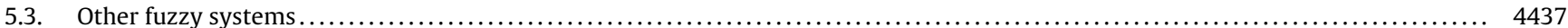

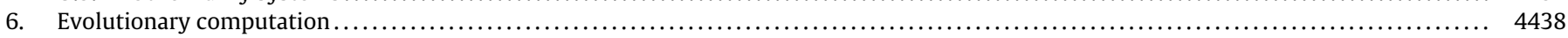

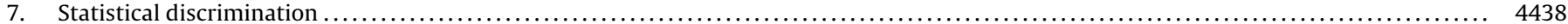

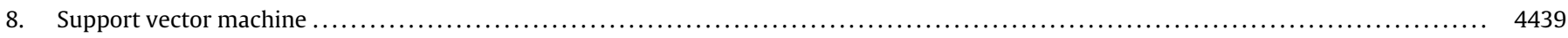

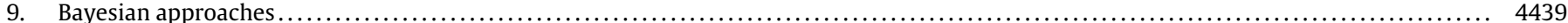

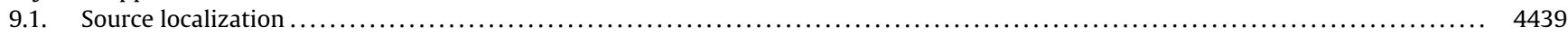

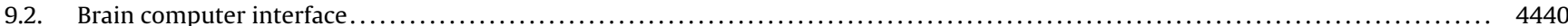

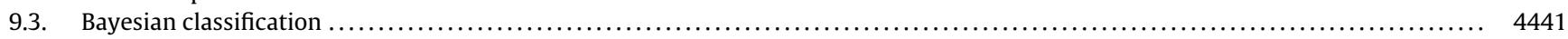

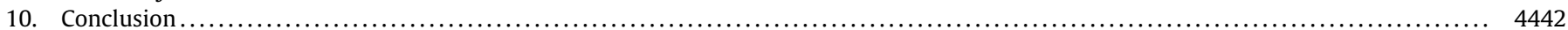

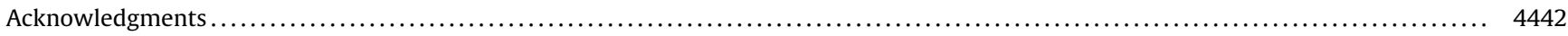

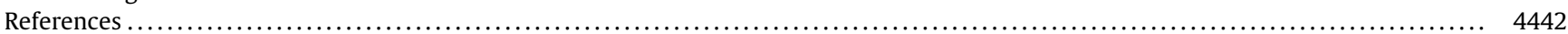

\footnotetext{
* Tel.: +919481901138.

E-mail address: kmajumdar@isibang.ac.in
} 


\section{Introduction}

In EEG analysis, most methods of analysis follow, explicitly or not, a pattern recognition approach $[1,2]$. These analyses have important applications in brain computer interface (BCI) [3-5], epilepsy research [6,7], sleep studies [8-10], psychotropic drug research and monitoring patients in critical condition in the ICUs $[11,12]$. However, automated analysis of EEG data is a huge challenge because of the volume of the data sets and dynamic nature of the signals with high temporal resolutions (in millisecond range). In case of the human scalp EEG signals this challenge has been further augmented by the introduction of high density EEG nets consisting of more than 300 channels [13] and with increasing sample frequency $(1000 \mathrm{~Hz}$ or more) of digitization by means of advanced technologies.

Human scalp EEG was born in 1920s when the German physician Hans Berger first measured traces of brain electrical activities on the scalp $[14,15]$. Since then the interpretation of patterns in the scalp EEG, in the most part, has remained a challenging issue. Synaptic activity in the pyramidal neurons ( $85 \%$ of excitatory human cortical neurons are of this type) is the principal source of scalp EEG [16] (p. 914). Modulatory dynamical actions of the neural ensembles, both at local and global scales, give rise to patterns in the scalp EEG $[17,18]$. With clever quantitative methods it is possible to measure (cognitive) task related integration [19-21] and differentiation [22] (in some sense) from even the single trial EEG signals.

The online epoch identification in human scalp EEG signals has a long history [23]. In this classic, the vision propounded for spatio-temporal data reduction and processing by soft computing approaches, like the Bayesian statistics, in order to bring down the computational loads to a manageable limit, are being largely followed even today [24]. For the sake of computational efficacy it is desirable to keep the analysis linear as far as possible. But, then comes the vital issue - are we not overlooking the nonlinear features? It has been argued in [25] that the advantage of a nonlinear analysis, at greater cost, of the multi-channel noisy scalp EEG data is rather marginal over the corresponding linear methods.

Since the early days of the BCI [26] the need for real time analysis of EEG and ERP has been felt. Linear analysis and soft computing techniques are the two most promising approaches in this regard. In contrast to classical approach of exact computation at a greater cost, which may be prohibitive for the complex problems like multidimensional EEG analysis, soft computing strives to achieve tangible results at reasonable cost by allowing inexactness and uncertainty to be part of the computational model. It includes neural networks, fuzzy logic, statistical discrimination, Bayesian inference and genetic algorithms. This list is of course not exhaustive, but would be sufficient for our purpose in this paper. Here we will be reviewing various soft computing techniques that have been followed for human scalp EEG/ERP processing. Such a review, even if non-exhaustive, would hopefully be useful for the research community.

Broadly speaking, EEG processing has two parts namely, (1) decomposing the complicated signal into simpler components (by FFT, wavelet transform, ICA, PCA, etc.) and (2) bunching those components together in search of specific structures in the data (the pattern recognition part). It is in the latter part, where almost all of these approaches are to deal with uncertainty and therefore they are soft computing approaches.

In the next two sections we will be briefly presenting a physiological overview of scalp EEG and dimensionality reduction of the data respectively. In Section 4 we will be reviewing neural network applications on human scalp EEG, in Section 5 fuzzy systems applications, in Section 6 applications of evolutionary computation, and in Sections 7-9 applications of statistical discrimination, support vector machine (SVM) and Bayesian inference, respectively. Not all these branches have found equal applications on human EEG. In this survey we have tried to be as exhaustive as we could, sacrificing the technical details, which can be found out in the references. This, we hope, will enhance the readability and usefulness of the paper.

\section{Cortical source of scalp EEG}

Excitatory postsynaptic potential (EPSP) at the apical dendritic trees of pyramidal neurons is the principal source of the scalp EEG $[15,16]$. When these neurons receive inputs through their apical dendrites EPSPs are generated in the apical dendritic tree. The apical dendritic membrane becomes transiently depolarized and consequently extracellularly electronegative with respect to the cell soma and the basal dendrites. This potential difference causes a current to flow through the volume conductor from the nonexcited membrane of the soma and basal dendrites to the apical dendritic tree sustaining the EPSPs $[1,15]$.

Some of the current takes the shortest route between the source and the sink by traveling within the dendritic trunk (primary current in blue in Fig. 1). Conservation of electric charges imposes that the current loop be closed with extracellular currents flowing even through the most distant part of the volume conductor (secondary current in red in Fig. 1). Intracellular currents are commonly called primary currents, while extracellular currents are known as secondary, return, or volume currents. With the spatial arrangement and the simultaneous activation of a large population of the cells, as shown in center of Fig. 1, contribute to the spatio-temporal superposition of the elemental activity of every cell, resulting in a current flow that generates detectable scalp EEG signals [15].

Both primary and secondary current contribute to scalp EEG. Macrocolumns of tens of thousands of synchronously activated large pyramidal cortical neurons are thus believed to be the principal sources of scalp EEG because of the coherent distribution of their large dendritic trunks locally oriented in parallel, and pointing perpendicularly to the cortical surface [27]. The currents associated with the EPSPs generated among their dendrites are believed to be at the source of most of the signals detected in MEG and EEG because they typically last longer than the rapidly firing action potentials traveling along the axons of excited neurons $[15,28]$.

\section{Dimensionality reduction}

Dimension of scalp EEG data at the preprocessing stage is calculated as number of channels $\times$ number of trials (e.g., the way the data representation is made [29]). For dense array EEG consisting of more than 100 channels, a recording session spanning through hundreds of trials each spanning through several seconds or minutes or even hours (in case of say, epilepsy monitoring) with a sample frequency of $1000 \mathrm{~Hz}$ or more, the amount of generated data may be of the order of tens or even hundreds of gigabytes. Without some kind of data reduction it would be impossible even to load the data set into the main memory of most modern day work stations. Dimensionality reduction can be done by selecting appropriate channels $[30,31]$ or time epochs or trials [32].

Dimension of EEG at the postprocessing stage is calculated usually in terms of the dimension of the feature space. Dimension reduction (also known as feature extraction) is achieved either by projection to a lower dimensional space or by selecting a subspace of the original one $[30,33]$. In [22] dimensionality reduction has been achieved by projecting EEG from all the channels into a single one dimensional time domain signal. More of it will be discussed in Section 7. 


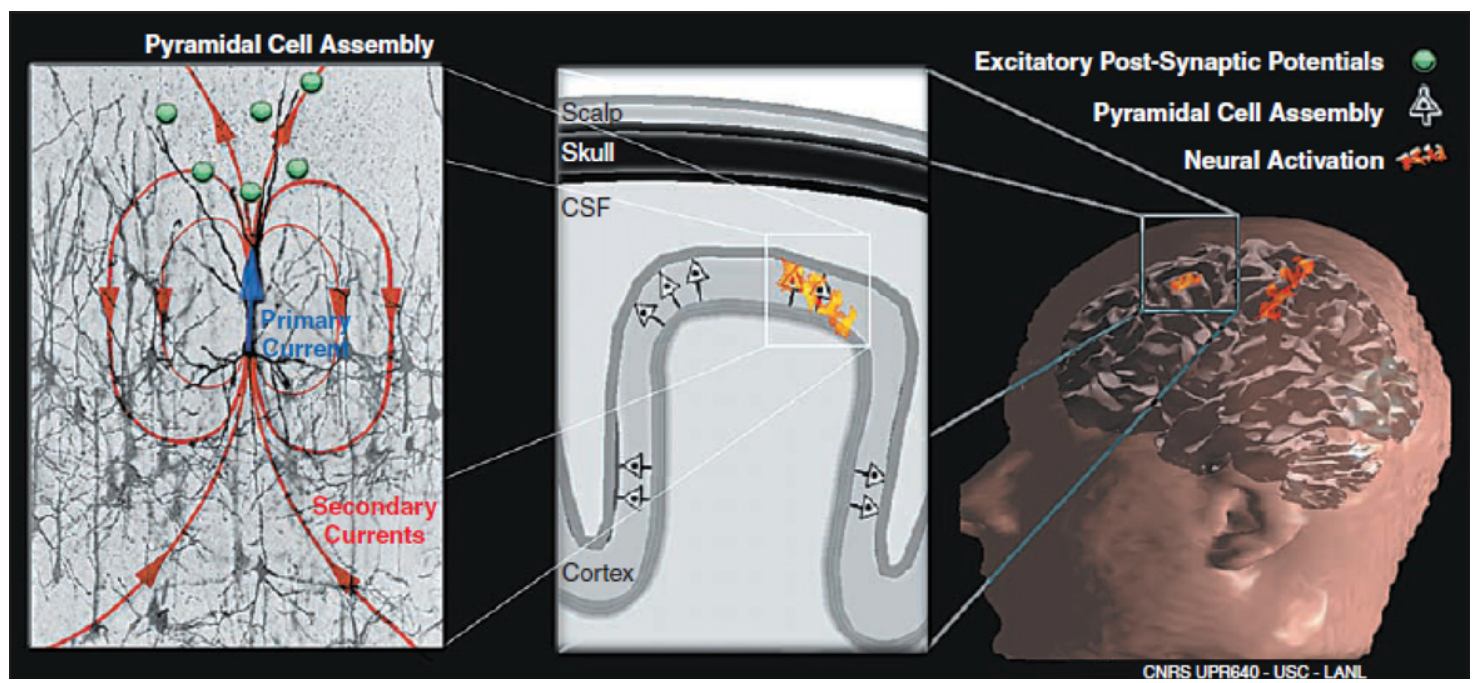

Fig. 1. Left: EPSPs are generated at the apical dendritic tree of a cortical pyramidal cell. Center: Large cortical pyramidal nerve cells are organized in macro-assemblies with their dendrites normally oriented to the local cortical surface. Right: Functional networks made of these cortical cell assemblies and distributed at possibly multiple brain locations are the main generators of EEG signals.

Adopted from [15]

\section{Neural networks}

This section will be organized in accordance with [34]. Low signal to noise ratio (SNR) in case of scalp EEG is a good reason for using ANN to process them [35].

\subsection{Artifact removal}

Eye blinks; movements of eyeballs and tongue; face, head and neck muscle contractions; cardiac rhythms; frequency of the alternating current supply to the equipment (steady state 50 or $60 \mathrm{~Hz}$ ) are the major sources of artifacts in scalp EEG (for a nice overview see Ref. [36]). Some of these may be avoided if the subject follows appropriate guidelines. For the others, automated artifact detection and removal techniques are the most practical solutions. When the patterns of artifacts are different from the patterns of evoked potential ANNs can theoretically be used to separate the artifacts out from the EEG. Some advancement in this direction has been reported in [37-46].

Various features of artifacts are extracted and fed into the input of an ANN to train it. At the end of the training, success rate of a radial basis function (RBF) network has been reported to be $75 \%$ in artifact detection [43].

\subsection{Source localization}

Interpretation of the clinical EEG almost always involves speculation as to the possible locations of the sources inside the brain that are responsible for the observed activity on the scalp [47]. For excellent reviews see $[15,48,49]$. However computational cost of most source localization algorithms is prohibitive. An error back propagation NN approach was first proposed to overcome this hurdle in case of dipole source localization [50]. In general dipole source localization problem is an optimization problem - to find optimum coordinate and orientation of dipoles, and hence suitable for being solved by ANN. It is possible to do away with computation intensive head models if there is sufficient input-output data to train the network.

A general ANN system for EEG source localization is illustrated in Fig. 2. According to [51], the number of neurons in the input layer is equal to the number of electrodes and the features at the input can be directly the values of the measured voltage. The network also consists of one or two hidden layers of $N$ neurons each and an output layer made up of six neurons, 3 for the coordinates and 3 for dipole components. In addition each hidden layer neuron is connected to the output layer with weights equal to one in order to permit a non-zero threshold of the activation function. Weights of inter connections are determined after the training phase where the neural network is trained with predetermined examples from forward modeling simulations [49]. Localization accuracy has been claimed to be less than $5 \%$ by various ANN approaches [34,35,50-57] and high accuracy in case of [58]. Clearly ANN approach is not very practical for distributed source models, where sources may consist of any subset of thousands of cortical mesh points [32].

\subsection{Sleep studies}

K-complexes are said to be the largest events in healthy human sleep EEG [59]. It is natural that ANN had been tried on them quite early with $90 \%$ success rate of identification and $8 \%$ false positive [60], also [61]. Sleep spindle identification by ANN also started getting attention at the same time $[60,62]$. A simple feed forward ANN was applied on sleep EEG even earlier [63]. 61-80\% accuracy was achieved in classifying seven different sleep stages in infant EEG (wake, movement, sleep stage 1 , sleep stage 2 , sleep stage 3/4, paradoxical sleep and artifacts) [64]. A pioneering study was undertaken to distinguish sleep EEG power spectrum patterns under the influence of different sleeping pills using ANN [65]. For a detailed review of early ANN applications on sleep studies see [66] (also see [34] for more references).

Use of ANN for automatic sleep stage scoring has been reported in [67] with an average $87.5 \%$ agreement with two human experts. A dominating trend in sleep EEG analysis has been - first to extract features (such as shape, frequency and power spectrum) by a suitable wavelet transform (in some cases Fourier transform [68]) and then using these features as input to an ANN $[67,69,70]$. Accuracy of recognition runs from as low as $44.44 \%$ [69] to around 95\% in [70]. Automatic recognition of alertness and drowsiness has been performed by three different ANNs with the best performance reported for the learning vector quantization (LVQ) network [71], which is $94.37 \pm 1.95 \%$ in agreement with the human experts.

\subsection{Epilepsy}

EEG analysis is an integral part of diagnosis and monitoring of epilepsy and it has a long history [72]. The effort for automatic 


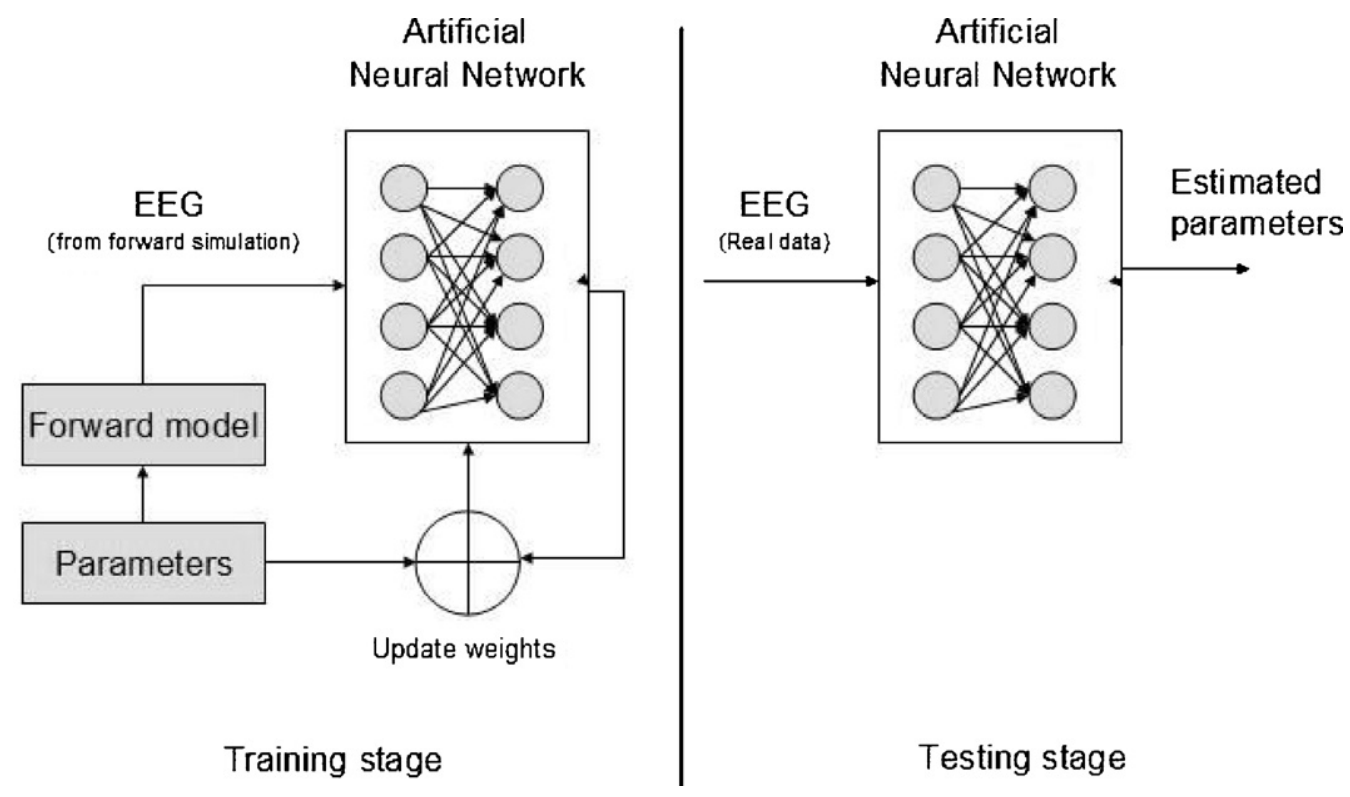

Adopted from [49].

Fig. 2. Block diagram for source localization by artificial neural networks (ANN).

detection of epileptic activities in prolonged EEG recordings is also quite old [73]. Neural networks started being used for epileptic seizure detection since early nineties $[74,75]$ followed by others [76-86]. In case of neonatal seizure detection by error back propagation NN the average detection rate is from $79.6 \%$ to $91 \%[85,86]$. Feed forward NN and quantum NN have been used to detect neonatal epileptic seizure with moderate specificity (little over $79 \%$ by both types of NN) [87]. Elman networks (ENs) have also been used for seizure detection [88]. These are a form of recurrent NN which have connections from their hidden layer back to a special copy layer. This means that the function learnt by the network can be based on the current inputs plus a record of the previous state(s) and outputs of the network. The results that EN yields are said to be the best with a single feature fed as the input. The overall reported detection accuracy is about $99.6 \%$ [88]. Recurrent NN based seizure prediction has been reported in [89]. For better performance of spike detection by NNs, preprocessing of the EEG has been emphasized in [90]. Recurrent $\mathrm{NN}$ has been used for seizure EEG classification in [91]. Scalp EEG of 418 epilepsy patients was classified with a multilayer perceptron (MLP), which matched with two human experts in $89.2 \%$ instances [92].

Let us conclude this subsection with a prophetic observation of Alan S. Gevins, "Brain electromagnetic signals can be quite useful for providing corroborating evidence about the presence of a seizure disorder and also for determining the site of seizure origin. Therefore, despite their limited clinical impact to date, efforts at automated "epileptiform" transient detection will undoubtedly continue" [93].

\subsection{Brain computer interface}

BCI started with the seminal paper of Farwell and Donchin [94]. Soon afterward NN was applied to classify the scalp EEG signals during right and left hand movements in the hope of predicting the side of movements before they occurred $[95,96]$. Power spectrum of extended $\alpha$-band $(5-16 \mathrm{~Hz})$ had been used to train and test an hybrid of K-means and back propagation NN to achieve a classification accuracy of $85-90 \%$ [97]. Cascade NN has been used for the same prediction purpose has shown widely varying results depending on the power spectrum of $\alpha$ EEG [98]. 91\% or more classification accuracies were achieved for mere left or right index finger movements discrimination by employing on ANN for each channel and selecting only the 'best' classification results $[99,100]$ ([100] also includes right foot movements in addition to the two mentioned earlier).

The performances of a back propagation ANN with four layers have been compared in [101] with two human investigators when both the ANN and the humans were engaged in classifying scalp EEG of six subjects during right middle finger extension tasks. For a cube rotation task in $\mathrm{BCI}$ an adaptive $\mathrm{NN}$ based algorithm has achieved a $68.3 \%$ classification accuracy in [102]. Imagined hand movement in four out of seven subjects is reported to be predictable with $80 \%$ accuracy in [103]. In a more recent study fast Fourier transform (FFT) based amplitudes of the EEG have been used as input to a multilayer NN with reported improved accuracy on test sets $80 \%$ or more [104]. FFT and NN based EEG classification of intention of right and left elbow movement has been reported in [105,106]. EEG classification of limb movement imagination by NN based on particle swarm optimization has been reported in [107].

\subsection{Other patterns}

Several studies have been reported pertaining to the analysis of evoked potential (EP) in the scalp EEG using NN [108-110]. Some of them are concerned about visual EPs [109-122], some about auditory EPs $[100,110,118,123-134]$ and some about somatosensory EPs [110,135-139]. For a fundamental treatment of use of NN in the analysis of event related potential (ERP) see Ref. [140].

Using EEG recordings several investigators have developed neural network based systems to assess the vigilance level of the subject under investigation [141-147]. In [147] a Levenberg-Marquardt (LM) multilayer perceptron (MLP) was used to classify EEG signals from 30 subjects for alertness (success rate 93.6\%), drowsiness (96.6\%) and sleep (90\%) (the LM network has been reported to be performing poorer than the LVQ in [71]). The input to the MLP was obtained by spectral analysis of the EEG through a discrete wavelet transform (DWT).

Analysis of maturation level of neonatal brains (28-112 weeks after birth) has been determined using NN on the EEG [148]. NN was used on EEG of 131 children aged between 4 and 16 years to 
detect possible abnormality in brain [149]. NN was applied on auditory EP of brainstem to detect hearing impairments in newborns [150]. Attention deficit hyper active (ADHA) disorder is a recognized problem in child psychiatry, in which NNs have been used on EEG to identify symptoms with good success $[151,152]$.

In certain neurological disorders EEG tends to be different from the normal. Tacitly using this fact NN based classification of disordered EEG with respect to the control has been achieved. This was done for headache and migraine [153-155], neuroophthalmological disorder [156], head injury [140], multiple sclerosis [39,157], schizophrenia [158-163], Alzheimer disease [164-167], Parkinson's disease [167], Huntington's disease [162,168-171], depression [161] and alcoholics [172,173]. Probabilistic NN has also been used for EEG classification in [174] with moderate success and slightly poorer performance than SVM, but with much better performance in [175]. For some clinical applications of NN on EEG see Section 4 of [176].

MLP and EN have been used on EEG to determine the depth of anesthesia during surgery with $99 \%$ success for the EN [177] (for a survey of applications of NN on EEG during anesthesia see Ref. [178]). EN has been shown to perform better on human visual evoked potential (VEP) than the k nearest neighbor (kNN) algorithm [179]. Continuous monitoring of brain state by means of NN application on EEG of the critically ill patients in the intensive care unit (ICU) has been reported in [12,180]. Use of NN on EEG under the effects of drugs (sedatives) in order to classify the effects due to different drugs has been reported in [181,182]. Classification of online scalp EEG by NN during three different mental tasks has been performed with $70 \%$ accuracy, but with only $5 \%$ mis-classification [183]. Convolutional NN has been used in BCI for classifying EEG during different activities with 95\% accuracy [184].

\section{Fuzzy logic}

Fuzzy logic based analysis of human scalp EEG started with the pioneering paper [8]. Fuzzy clustering and neuro-fuzzy techniques have remained the most notable methodologies in this regard.

\subsection{Fuzzy clustering}

Cluster analysis is based on partitioning a collection of data points into a number of subgroups, where the objects inside a cluster (a subgroup) show a certain degree of closeness or similarity. Hard clustering assigns each data point (feature vector) to one and only one of the clusters, with a degree of membership equal to one, assuming well defined boundaries between the clusters. This model often does not reflect the description of real data, where boundaries between subgroups might be fuzzy, and where a more nuanced description of the object's affinity to the specific cluster is required [10]. In case of human EEG this was first utilized in [8] (before this fuzzy clustering was applied on sleep EEG of chimpanzee [185]). An efficient human sleep EEG data classification has been reported in [10] by means of unsupervised fuzzy partition-optimal number of classes (UFP-ONC), which is a combination of fuzzy k-means (FKM) algorithm [186] and fuzzy maximum likelihood estimation. This has been able to decompose the sleep EEG from a single subject into optimum number of distinct classes, which has been treated as a priori unknown $[10,187,188]$.

A different fuzzy clustering algorithm was used in [189] for EP identification in low signal to noise ratio (SNR) EEG. In this FKM algorithm has been applied with the number of clusters determined by the criterion proposed in [190]. Trials with prominent (same) EP were grouped together using fuzzy clustering before being averaged for extraction of the EP. Single instances of EP have been reported to be classified up to 95\% accuracy. FKM clustering (also known as fuzzy c-means clustering) was used in conjunction with an ANN to classify epileptic spikes (ES) in scalp EEG [191]. However the performance is not very impressive.

Fuzzy if-then rule-based online classification of a single subject's EEG signal during pain and no pain experiences has been reported in [192] with only 64\% overall classification accuracy, which is slightly poorer than the corresponding hidden Markov model (HMM) classification studied on the same data set. A fuzzy classification technique for epilepsy risk level has been proposed in [193].

\subsection{Neuro-fuzzy techniques}

Combination of NN and fuzzy logic gives a powerful soft computing methodology, which has been applied on human EEG with mixed success. In one of the first applications auditory evoked potential (AEP) from the EEG of a patient under anesthesia was analyzed by an NN. The output of the NN was utilized as input to a fuzzy if-then rule-based controller, which controlled the dosage of the anesthetic drug. The performance was graphically compared with a trained anesthetist during a real surgery [194].

About $88.2 \%$ infant sleep-wake stage classification on the test EEG data has been achieved by ANFIS-based classifier [195] (Fig. 3). The architecture is in Fig. 3. Layer 1 is the fuzzification layer. $X_{1}, X_{2}$, and $X_{3}$ are three of the input variables, each with two associated fuzzy concepts $\left(A_{i}\right.$ and $\left.B_{i}\right)$. Layer 2 generates all the possible rules of the form IF $X_{1}$ is $A_{1}$ and $X_{2}$ is $B_{2}$ and $X_{3}$ is $A_{3}$, with a T-norm operator $(\cdot)$, considering one fuzzy concept per input variable. The output of layer 2 is a strength parameter for each of the rules. Each node at layer 3 performs a linear combination of the rules and uses a sigmoidal function to determine the degree of belonging of the input pattern to each class $\left(C_{1}, C_{2}, C_{3}\right)$. In another study ANFIS classifiers were used on features extracted from EEG by wavelet transformations (WTs) for classification pertaining to five different classes with a total accuracy of $98.68 \%$ [196]. WT on EEG followed by ANFIS could classify normal subjects from epileptic patients with $93.7 \%$ and $94.3 \%$ respectively, which is slightly higher than that achieved by an MLP [84]. WT followed by ANFIS has been used to analyze EEG pertaining to left and right hand movements [197], state of alertness [198]. Neuro-fuzzy NN has been used to determine the states of fatigue or alertness in drivers [199]. EEG feature extraction by Lyapunov exponent followed by ANFIS classification was used to detect changes in the signal [200]. A comparative study of neuro-fuzzy classifiers with some other classification methods is also available [201]. For a comprehensive treatment of the subject see Ref. [202].

Combining adapted resonance theory (ART) NN with fuzzy logic, fuzzy ARTMAP NN was created [203], which has found several applications in human EEG processing [169,204-208], often with classification success rate of $90 \%$ or above. Very recently a faster self-organizing fuzzy neural network has been applied in $\mathrm{BCI}$ with up to $70 \%$ processing time reduction [209].

\subsection{Other fuzzy systems}

After extracting features from EEG by DWT fuzzy SVM (FSVM) has been applied for the classification [210]. However FSVM is reported to have given poor results on classification of schizophrenic EEG from the control subjects [211]. Features extracted from EEG using wavelet packet have been sorted by fuzzy logic for optimum performance [212]. Fuzzy if-then rules have been used on features extracted by time frequency analysis of EEG in order to determine the depth of anesthesia on 22 patients [213]. Fuzzy rule based detection of $\alpha$-band activity has been proposed in [214]. EEG based use of a fuzzy controller has been proposed to administer anesthesia in [215]. 


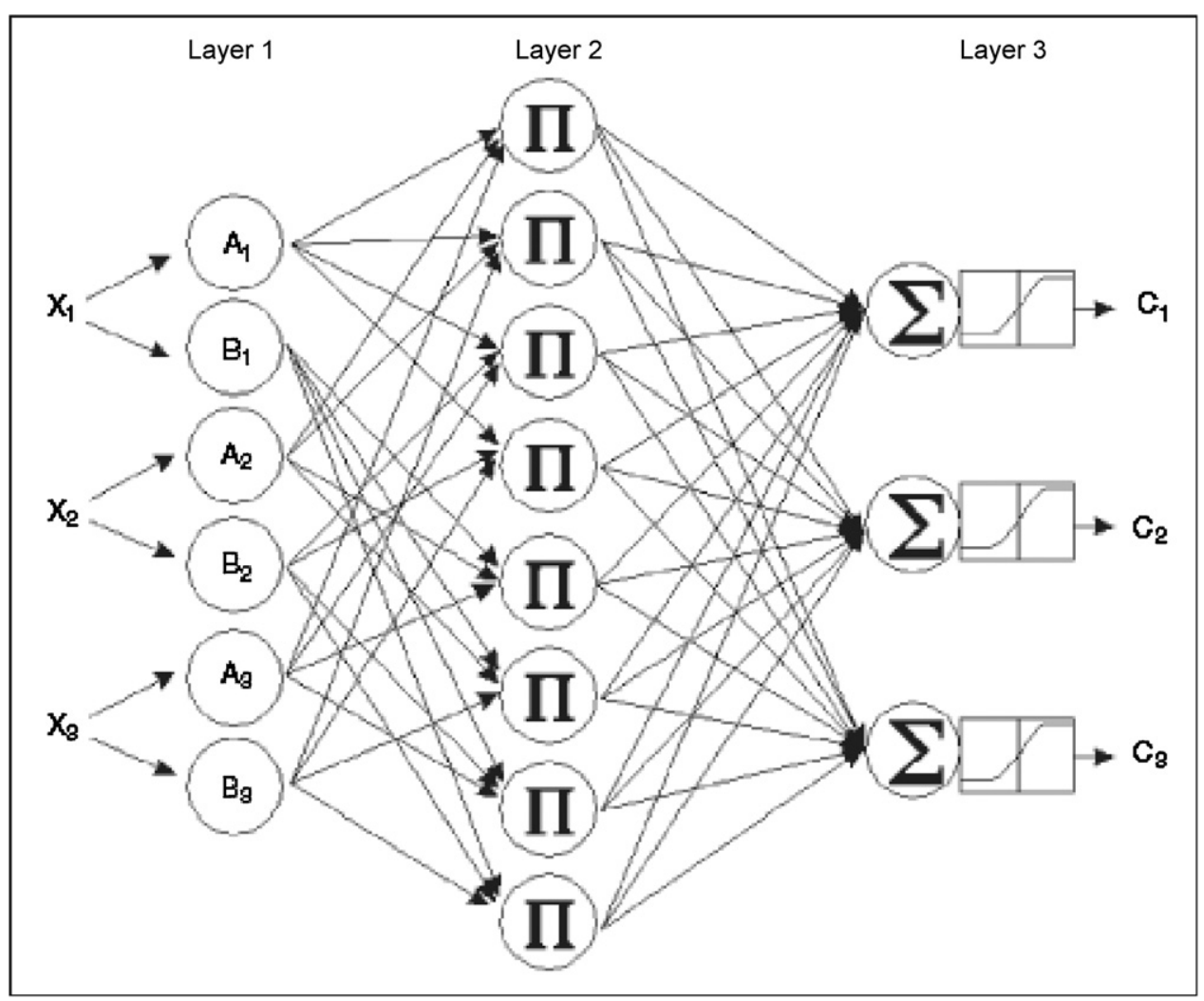

Adopted from [195].

Fig. 3. A simple adaptive neuro-fuzzy inference system (ANFIS) for infant sleep-wake stage classification.

\section{Evolutionary computation}

Signals in medicine, such as EEG, processing is subject to several important constraints. First, the number of signals to be processed is high, and often tightly interdependent. Second, signals are unique, in the sense that the circumstances under which they were obtained are normally not repeatable. Third, given the characteristics of their sources, medical signals are often very noisy. Finally, in some cases information about the signals is required in real time in order to take crucial decisions [216]. Genetic algorithm (GA) was applied on EEG during different mental tasks in order to classify them in task specific categories. The goal was achieved with 76\% accuracy [25]. Genetic programming (GP) has been applied on human scalp EEG for epileptic pattern recognition [217] with success rate of $93 \%$ or more (in intracranial EEG seizure precursor features have been detected by GP in [218]). GP has been used for normal EEG classification in [219]. Epilepsy risk assessment with GA has been done in $[220,221]$.

\section{Statistical discrimination}

Statistical discriminants are standard tools for classification of multidimensional patterns (for a general introduction see Ref. [222]). Their need in human scalp EEG classification has long been felt [23]. Using them on scalp EEG, classification of dyslexia patients was performed in [223]. EEG of mild head injury patients was classified (with respect to a group of normal control subjects) by statistical discriminants with more that $90 \%$ accuracy in [224,225]. [226] presents a review of classification of scalp EEG by discriminators in case of traumatic brain injury. Discriminants have been used to classify EEG belonging to subjects with neuropsychiatric disorders [227]. Unfortunately, very little detail is available of the discriminators implemented in [224-227].
Scalp EEG of normal human subjects has been classified during rapid serial visual presentation (RSVP) of 'interesting' and 'uninteresting' scenes by statistical discrminants [22,228,229]. Discriminant analysis has been performed in single trials on the weighted sum of all the scalp channels, where the optimum weight has been selected by fine tuning a logistic regression (LR) function (for a nice exposition of LR see Ref. [230]) with the help of gradient descent method [22]. Then normalized projection of signal from each channel on this average is calculated. Intensity of this projection is used to classify signals between interesting $(91.8 \%$ classification accuracy) and uninteresting scenes (98.3\% classification accuracy) [228].

Although LR is more robust, it is a less efficient classifier and takes more resources to compute compared to the normal statistical discriminators [231]. A study was undertaken to compare performance between Fisher's discriminant (FD, see [232,233] for description) and LR on the scalp EEG of three subjects (two males and one female, mean age thirty years, all of them left handed). They did not have any known neurological or vision disorder. The data was collected using 256 channel Hydrocell Geodesic Sensor Net (Electrical Geodesics, Inc., Eugene, OR) during a series of RSVP tasks at a rate of 3 grey level satellite images per second [234]. The analysis was performed on single trials. LR turned out to be good in identifying target, but poor in identifying non-target data (Table 1).

Table 1

Average performance of LR vis-à-vis FD on the EEG of three subjects during RSVP (3 images per second) of three different targets vs. non-target. ROC area means the area under the receiver operator characteristic (ROC) curve.

\begin{tabular}{lll}
\hline & LR & FD \\
\hline Target & 0.9752 & 0.7601 \\
Non-target & 0.5768 & 0.8770 \\
ROC area & 0.9311 & 0.8700 \\
\hline
\end{tabular}


Table 2

Average performance of LR vis-à-vis FD on the EEG of three subjects during RSVP ( 3 images per second) of target tank and target truck in different sessions (each consisting of about 300 trials) in each of which only one type of target images are mixed with non-target images roughly at 1:4 ratio.

\begin{tabular}{lll}
\hline & LR & FD \\
\hline Tank & 0.6186 & 0.9858 \\
Truck & 0.7623 & 0.9751 \\
ROC area & 0.7067 & 0.9939 \\
\hline
\end{tabular}

On the other hand FD was poor in identifying target data, but much better in identifying non-target data (Table 1). FD was also good in separating various pairs of target EEG data (see Table 2 for an example). The general conclusion was that there is no particular discriminator uniformly suitable for all types of EEG data. Different discriminators perform differently on different data sets [234]. FD was used on EEG after feature extraction by a combination of continuous WT and student $t$-statistic with the best classification accuracy in the $2003 \mathrm{BCI}$ competition [235]. FD was used for random classification of EEG channels for $\mathrm{BCI}$ in [236] with a very moderate accuracy of $56.66 \%$. A comparison of FD and two of its variants with SVM and k nearest neighbor (kNN) algorithm on EEG data before onset of finger movements appears in [237]. The outcomes are presented in Table 3. For a review of applications of linear discriminant analysis in BCI research see Ref. [238].

LR has been compared with NN on seizure EEG data [83]. Classification accuracy of two different MLPs has been reported to be more than $91 \%$ compared to $89 \%$ for the LR. Superior performance of NN over LR has been reported in $[239,240]$. On an average LR had performed better on the single trial EEG than a conventional spatial pattern (CSP) based classifier [241].

A statistical discriminant was used to classify EEG signals belonging to schizophrenic patients for negative and positive features associated with the symptoms. $78 \%$ classification accuracy for schizophrenia was achieved on a test data set (disjoint from the training data) with $85 \%$ specificity [242]. Quadratic discriminant function was applied on EEG of 33 subjects to classify among different tasks with 93\% accuracy for the training data and 85\% accuracy for the testing data [243].

\section{Support vector machine}

Despite greater difficulty in implementation and longer running time on test data compared to the NN and linear discriminants, SVM has become a popular classification algorithm for the EEG for its usually higher classification accuracy compared to the former. For an excellent tutorial on SVM see Ref. [244]. The primary motivation behind SVM is to directly deal with the objective of generalization from training data to testing data with minimization of error and complexity of the learning algorithm [25]. Table 3 shows superior performance of SVM on EEG data. A recent study on classification (vis-à-vis a human expert) of neonatal EEG of six infants has shown that SVM has outperformed the FD and NN (Fig. 4) [245].

Table 3

Test set error ( \pm std) for classification at $120 \mathrm{~ms}$ before keystroke. 'mc' refers to the 21 channels over (sensori) motor cortex, 'all' refers to all 27 channels. RFD and SFD stand for regularized and sparse FD respectively. ch stands for channel.

\begin{tabular}{rlrllll}
\hline Filter & ch's & \multicolumn{1}{l}{ FD } & \multicolumn{1}{l}{ RPD } & \multicolumn{1}{l}{ SFD } & \multicolumn{1}{l}{ SVM } & \multicolumn{1}{l}{ k-NN } \\
\hline$<5 \mathrm{~Hz}$ & mc & $3.7 \pm 2.6$ & $3.3 \pm 2.2$ & $3.3 \pm 2.3$ & $3.2 \pm 2.5$ & $21.6 . \pm 4.9$ \\
$<5 \mathrm{~Hz}$ & all & $3.3 \pm 2.5$ & $3.1 \pm 2.5$ & $3.4 \pm 2.7$ & $3.6 \pm 2.5$ & $23.1 \pm 5.8$ \\
None & mc & $18.1 \pm 4.8$ & $7.0 \pm 4.1$ & $6.4 \pm 3.4$ & $8.5 \pm 4.3$ & $29.6 \pm 5.9$ \\
None & all & $29.3 \pm 6.1$ & $7.5 \pm 3.8$ & $7.0 \pm 3.9$ & $9.8 \pm 4.4$ & $32.2 \pm 6.8$ \\
\hline
\end{tabular}

Reproduced from [237].
Artifacts such as, eye blink potential and electrocardiogram (ECG) have been removed from EEG using SVM [246]. A nonlinear SVM was applied to distinguish P300 EEG epochs from the other EEG signals during visualization of different words with $84.5 \%$ accuracy [247]. In another application on P300 based speller classification a self-supervised SVM has been applied to reduce the training efforts [248]. In [249] average P300 classification accuracy by SVM has been reported to be above $95 \%$. Superior performance of SVM than linear discriminant analysis and $\mathrm{k}$ nearest neighbor classifier on the EEG of five subjects during limb and tongue movements has been reported in [250]. Better performance of SVM over PNN and multilayer PNN in EEG classification has been reported in [174]. 90\% accuracy in EEG classification by SVM during left, right finger movements has been reported in [251]. SVM as part of ensemble classification for EEG has been considered in [252].

\section{Bayesian approaches}

\subsection{Source localization}

If $J_{n}$ is an $n$-dimensional vector of cortical sources and $M_{p}$ be a $p$-dimensional measurement of scalp EEG, where $n$ and $p$ are number of sources and number of channels respectively. Then by Bayes theorem

$p\left(J_{n} \mid M_{p}\right)=\frac{p\left(M_{p} \mid J_{n}\right) p\left(J_{n}\right)}{p\left(M_{p}\right)}$,

where $p(A \mid B)$ is the conditional probability of event $A$, given event B. $p\left(M_{p}\right)$ is constant. The configuration $J_{n}$ for which maximum of (1) will be achieved is the most probable source of $M_{p}$. This is called maximum a posteriori (MAP) estimation [253,254]. We can write $p\left(J_{n} \mid M_{p}\right)=(1 / Z) \exp \left(-U\left(J_{n}\right)\right), Z$ is a normalization constant and $U$ is an 'energy' function. Taking logarithm and treating $p\left(M_{p}\right)$ as a constant throughout, we can write $U\left(J_{n}\right)=U_{1}\left(J_{n}\right)+\lambda U_{2}\left(J_{n}\right)$, where $\lambda$ is a constant, and $U_{1}\left(J_{n}\right)$ and $U_{2}\left(J_{n}\right)$ are associated with $p\left(M_{p} U_{n}\right)$ and $p\left(J_{n}\right)$ respectively. $U_{1}\left(J_{n}\right)=\left\|M_{p}-G J_{n}\right\|$, where $G$ is a $p \times n$ mixing matrix made out of the head model of the subject. $U_{2}\left(J_{n}\right)=U_{s}\left(J_{n}\right)+U_{t}\left(J_{n}\right)$, where $U_{s}$ and $U_{t}$ are associated with spatial and temporal priors respectively. Five different algorithms were used in [253] to calculate the MAP in (1). In [254] Bayesian MAP has been used to estimate error in the reconstructed sources. Unlike [253], in [254] the prior has been modeled by chi-square distribution function.

Bayesian model averaging has been applied for EEG source localization, which determined the posterior probability of the sources according to the best available model [255]. Repeated Bayesian estimation of maximum entropy of EEG has been used for the source localization [256]. A hybrid of two source models - equivalent current dipole (ECD) model and distributed source (DS) model has been proposed in [257]. Source reconstruction has been performed under suitable spatial and temporal constraints estimated by Bayesian method. EEG source reconstruction was done in [258] according to both ECD and DS models by formulating the inverse problem as Bayesian inference, like in (1). The forward model was constructed by Markov chain Monte Carlo (MCMC) method.

A general framework for Bayesian interpretation of brain images has been proposed in [259]. It has been applied for EEG source localization in [24]. Source localization has been performed with no prior, accurate prior, inaccurate prior, and a mixture of accurate and inaccurate prior. Results obtained on spherical head model with simulated data under different SNR and three different inverse methods subject to Bayesian expectation maximization. A result is shown in Fig. 5. Automatic selection of multiple cortical sources with compact support in a DS model has been achieved in [260] with a new application of [259]. In another application evoked and induced responses with respect to a stimulus has been 


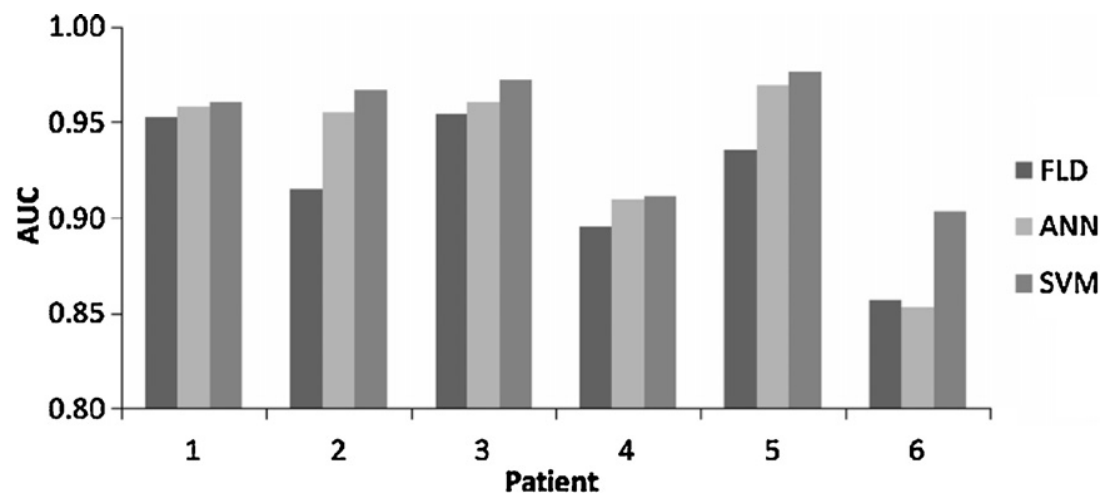

Fig. 4. Six infants shown in abscissa. Performance measure is given by area under curve (AUC) of the ROC curve. FLD stands for Fisher's linear discriminant. Adopted from [245].

reconstructed in the cortical surface from scalp EEG data [261]. Bayesian learning has been utilized to identify common sources of EEG and fMRI (functional Magnetic Resonance Imaging) in human subjects in [262].

\subsection{Brain computer interface}

Bayesian NN was used on EEG to detect imagined finger movements in [263] with a typical accuracy of $75 \%$. A real time $\mathrm{BCl}$ was designed with minimum training and using only one channel EEG data with $86.5 \pm 6.9 \%$ classification accuracy for cursor movement task in [264]. The minimum training was possible under a
Bayesian paradigm. A Bayesian inference scheme to predict continuous cursor movement has been proposed in [265,266]. A dynamic Bayesian network (DBN) model has been used to predict the movement intention, where the DBN has learned from the EEG and EMG (electromyogram) [267].

A comparative study among Bayesian graphical network, neural network, Bayesian quadratic, Fisher linear and hidden Markov model as classifiers of EEG for BCI applications has been presented in [268] (Table 4). BGN and Bayesian quadratic classifier seem to have performed better than others. Bayesian linear discriminant analysis has been applied for EEG classification in BCI in [269], with a superior performance than SVM and linear discriminant.
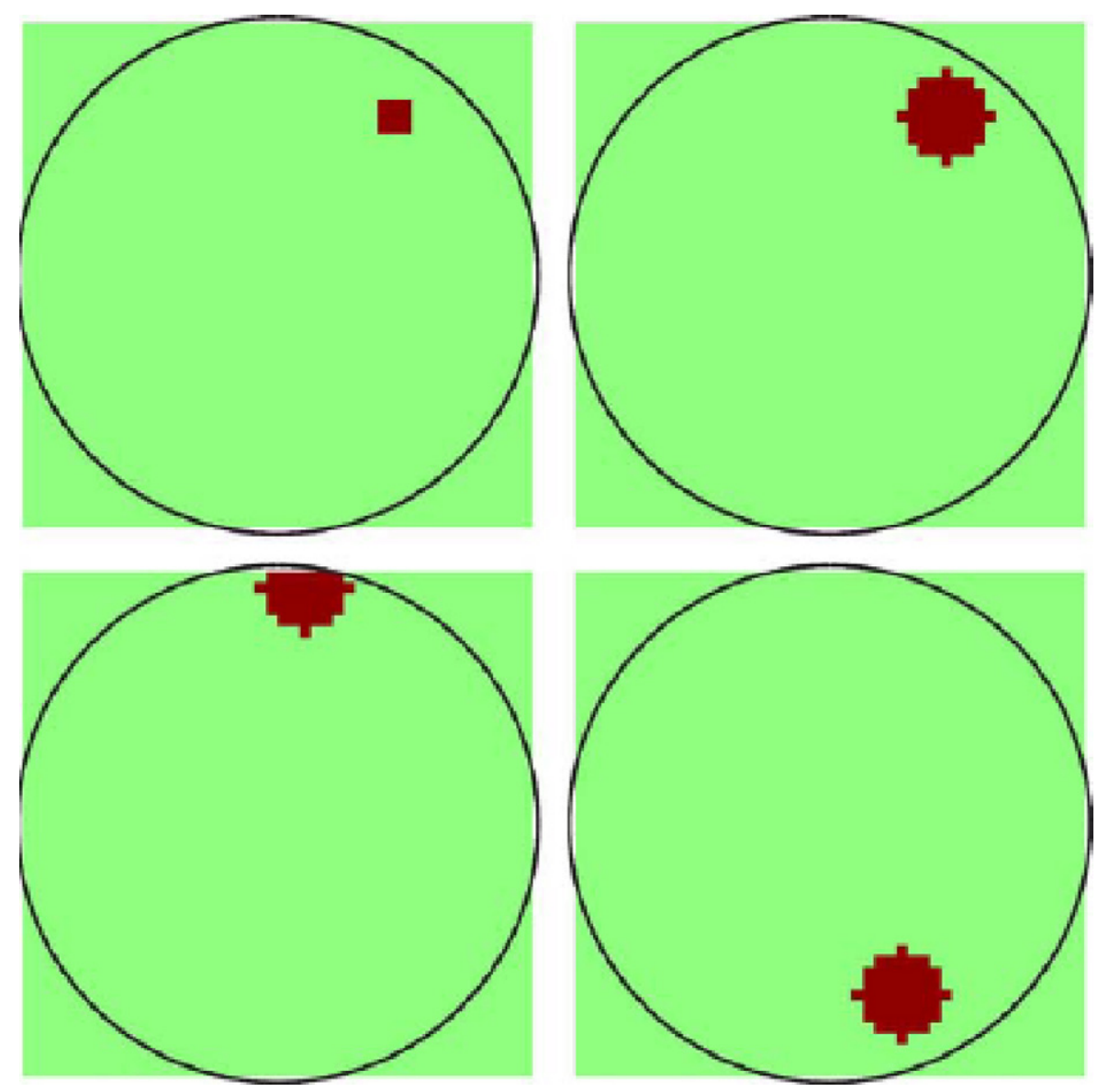

Fig. 5. Example of a source used in the simulations (top left) with the corresponding accurate location priors (top right), as well as inaccurate location priors (close, bottom left, and distant, bottom right).

Adopted from [258]. 
Table 4

The Bayesian graphical network (BGN), neural network, Bayesian quadratic classifier, Fisher linear and hidden Markov model (HMM) are compared for classification of binary combinations of five mental tasks. The results in the table are averaged over ten different possible binary combinations of mental tasks.

\begin{tabular}{|c|c|c|c|c|c|}
\hline Subject & BGN & Neural network & Bayesian & Fisher lilies & HMM \\
\hline 1 & $94.07 \pm 2.2$ & $92.48 \pm 2.9$ & $93.78 \pm 2.8$ & $91.15 \pm 2.7$ & $70.18 \pm 8.8$ \\
\hline \multirow[t]{2}{*}{3} & $87.43 \pm 3.9$ & $85.04 \pm 4.3$ & $89.22 \pm 3.5$ & $82.77 \pm 4.1$ & $64.10 \pm 9.1$ \\
\hline & $82.48 \pm 2.8$ & $82.61 \pm 3.0$ & $86.58 \pm 3.4$ & $81.79 \pm 3.1$ & $62.43 \pm 7.8$ \\
\hline 6 & $90.31 \pm \pm 2.7$ & $89.39 \pm 3.1$ & $92.49 \pm 3.2$ & $90.38 \pm 3.1$ & $64.61 \pm 8.3$ \\
\hline Means & $88.57 \pm .3 .0$ & $87.38 \pm 3.4$ & $90.51 \pm .3 .2$ & $86.63 \pm 3.3$ & $65.33 \pm 8.5$ \\
\hline
\end{tabular}

Reproduced from [268].

\subsection{Bayesian classification}

There are two standard approaches to EEG classification - discriminative and generative. Bayesian classification falls under the generative class. For a nice overview see Ref. [270]. In a generative approach, we define a model for generating data $V$ belonging to particular mental task $c \in\{1, \ldots, C\}$ in terms of a distribution $p(V \mid c)$. Here, $V$ will correspond to a time-series of multi-channel EEG recordings, possibly preprocessed. The class $c$ will be one of the mental tasks. For each class $c$, we train a separate model $p(V \mid c)$, with associated parameters $\Theta_{c}$, by maximizing the likelihood of the observed signals for that class. We then use Bayes rule to assign a novel test signal $V^{*}$ to a certain class $c$ according to: $p\left(c \mid V^{*}\right)=\frac{p\left(V^{*} \mid c\right) p(c)}{p\left(V^{*}\right)}$. That model $c$ with the highest posterior probability $p\left(c \mid V^{*}\right)$ is designated the predicted class [270].

Input output Hidden Markov model (IOHMM, see Ref. [271], and Fig. 7(d) for the architecture) based classification of EEG, which is a special case of Bayesian classification, has been applied in $\mathrm{BCI}$ [270]. IOHMM has performed better than HMM, Gaussian mixture model (GMM) and MLP with reduced classification error rate. HMM was applied on whole night EEG of nine subjects for sleep stage classification with accuracy ranging from 26\% (rapid eye movement sleep) to $86 \%$ (wake stage) [272]. To overcome the problem of nonstationarity in EEG signals HMM has been introduced, which then according to the scheme presented in Fig. 6 determines if the movement intention is on left or right by evaluating the expression $\operatorname{MAX}\left(P_{P}\left(V \mid \mathrm{HMM}_{\mathrm{L}}\right), P_{P}\left(V \mid \mathrm{HMM}_{\mathrm{R}}\right)\right)$ [273]. The online classification

a

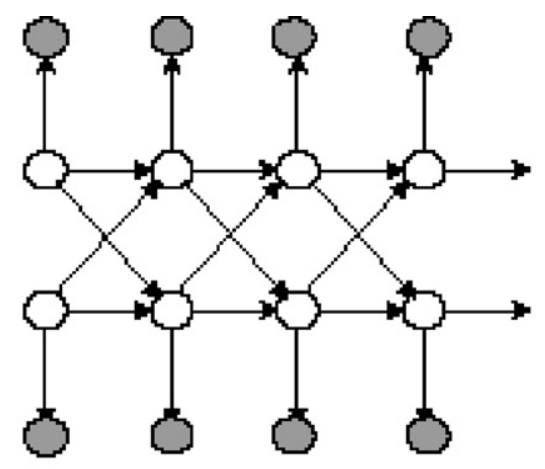

C

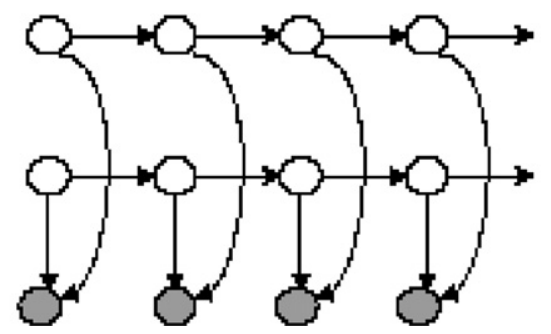

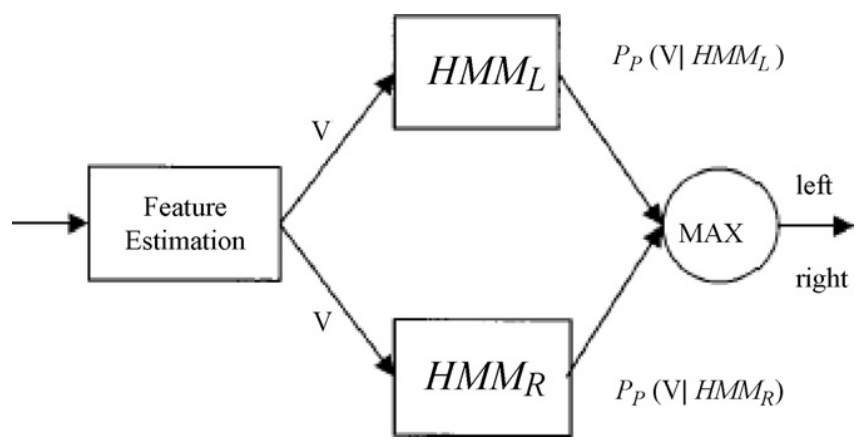

Fig. 6. $\mathrm{BCl}$ system comprising $\mathrm{HMM}_{\mathrm{L}}$ for left movement feature selection and $\mathrm{HMM}_{R}$ that for the right.

Adopted from [273].

rate occurring in four healthy subjects varied between $75 \%$ and $95 \%$ [274]. For theory and some applications of HMM see Refs. [275,276]. HMM on EEG was used to classify arousal and sleep states in [277]. Various HMM architectures have been shown in Fig. 7. A comparative study of their performances on human EEG data has been presented in [278]. HMM along with Principal Component Analysis (PCA) and SVM has been applied on EEG to classify left right movement in [279].

Kernel PCA and HMM are combined to identify mental fatigue features in EEG in [280] with a classification accuracy of $88 \%$. The

b

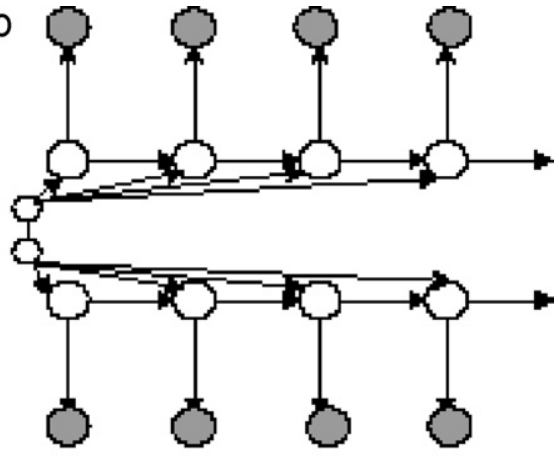

d

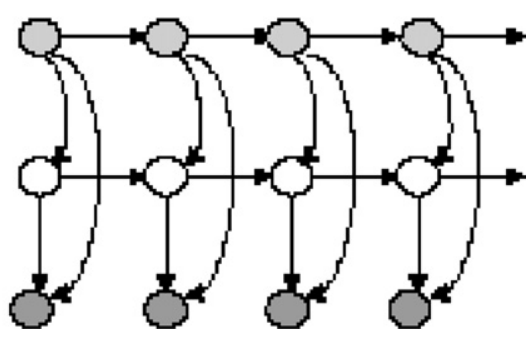

Fig. 7. Various HMM architectures. The empty circles are the hidden states and the shaded ones are observation nodes, the lightly shaded ones (in d) are input nodes. (a) Standard coupled HMMs; (b) event coupled HMMs; (c) factorial HMMs; (d) input-output HMM.

Adopted from [278]. 
signal was collected during prolonged viewing at visual display terminal (VDT). HMM has also found applications in designing and validating seizure prediction algorithms [281].

\section{Conclusion}

EEG signals are multidimensional, nonstationary (i.e., statistical properties are not invariant in time), time domain biological signals, which are not reproducible. It is supposed to contain information about what is going on in the ensemble of excitatory pyramidal neuron level, at millisecond temporal resolution scale. Since scalp EEG contains considerable amount of noise and artifacts, and exactly where it is coming from is poorly determined, extracting information from it is extremely challenging. So far the two major paradigms used to understand scalp EEG are - segregation (classification, clustering, etc.) and integration (synchronization, coherence, etc.), both of which are computation intensive. The current explosion of interest in $\mathrm{BCI}$, on the other hand, underscores the need of online processing. This is a compelling reason for the popularity of soft computing algorithms in human scalp EEG processing.

The class of soft computing algorithms is not precisely defined. Any algorithm which employs inexact or approximate calculations may fall under this category. But for this paper by a soft computing algorithm we have understood any technique falling under one or more of the following categories: neural networks, fuzzy logic, evolutionary computation, statistical discrimination, support vector machine and Bayesian approaches. From a literature survey it appears that neural networks and Bayesian approaches are the two most popular choices in EEG processing. Linear statistical discriminants are easier to implement, but support vector machines give (many a times marginally) better classification accuracy. It is a choice between cost of implementation and significance of difference in performance. The popularity of fuzzy logic and genetic programming based techniques in human scalp EEG processing are yet to catch up with the remaining four. In general there is no 'good' or 'bad' technique in EEG processing. An 'efficient' technique is to be chosen depending on the data set and processing goal. In this sense, along with more 'exact' computing, the soft computing technique paradigms discussed in this paper constitute major human scalp EEG processing methodologies for the last three decades.

\section{Acknowledgments}

The author likes to thank the library of the Florida Atlantic University, Boca Raton, Florida for allowing online access to their electronic archive as a former staff without, which it would not have been possible to write this article. The author is also thankful to his present employer, the Indian Statistical Institute for liberal work conditions. The data analysis was done when the author was working jointly in the Electrical Geodesics Inc. and the University of Oregon, Eugene, OR, USA under a DARPA grant for the "Neurotechnology for intelligence analysts" project.

\section{References}

[1] F. Lopes da Silva, Computer-assisted EEG diagnosis: pattern recognition and brain mapping, in: E. Niedermeyer, F.H. Lopes da Silva (Eds.), Electroencephalography: Basic Principles, Clinical Applications, and Related Fields, 5th ed., Lippincott Williams \& Wilkins, London, 2004, pp. 1233-1264.

[2] A.S. Gevins, Pattern recognition of brain electrical potentials, IEEE Trans. Pattern Anal. Mach. Intell. 2 (5) (1980) 383-404.

[3] J.R. Wolpaw, N. Birbaumer, W.J. Heetderks, D.J. McFarland, P.H. Peckham, G. Schalk, E. Donchin, L.A. Quatrano, C.J. Robinson, T.M. Vaughan, Brain computer interface technology: a review of the first international meeting, IEEE Trans. Rehabil. Eng. 8 (June (2)) (2000) 164-173.

[4] T.M. Vaughan, W.J. Heetderks, L.J. Trejo, W.Z. Rymer, M. Weinrich, M.M. Moore, A. Kubler, B.H. Dobkin, N. Birbaumer, E. Donchin, E.W. Wolpaw, J.R
Wolpaw, Brain computer interface technology: a review of the second international meeting, IEEE Trans. Neural Syst. Rehabil. Eng. 11 (June (2)) (2003) 94-109.

[5] M.A. Lebedev, M.A. Nicoleis, Brain-machine interface: past, present and future, Trends Neurosci. 29 (September (9)) (2006) 536-546.

[6] H. Witte, L.D. Iasemidis, B. Litt, Special issue on epileptic seizure prediction, IEEE Trans. Biomed. Eng. 50 (May (5)) (2003) 537-538.

[7] J. Engel Jr., T.A. Pedley, Epilepsy: A Comprehensive Text Book, vols. 1 and 2, 2nd ed., Lippincott Williams \& Wilkins, London, 2007.

[8] A. Kumar, A real-time system for pattern recognition of human sleep stages by fuzzy system analysis, Pattern Recogn. 9 (1) (1977) 43-46.

[9] B.H. Jansen, W.K. Cheng, Classification of sleep patterns by means of Markov modelling and correspondence analysis, IEEE Trans. Pattern Anal. Mach. Intell. 9 (September (5)) (1987) 707-710.

[10] I. Gath, A.V. Geva, optimal fuzzy clustering, IEEE Trans. Pattern Anal. Mach. Intell. 11 (July (7)) (1989) 773-780.

[11] K.G. Jordan, Continuous EEG and evoked potential monitoring in the neuroscience care unit, J. Clin. Neurophysiol. 10 (1993) 445-475.

[12] R. Agarwal, J. Gotman, D. Flanagan, B. Rosenblatt, Automatic EEG analysis during long-term monitoring in ICU, Electroencephalogr. Clin. Neurophysiol. 107 (July (1)) (1998) 44-58.

[13] R. Oostenveld, P. Praamstra, The five percent electrode system for highresolution EEG and ERP measurements, Clin. Neurophysiol. 112 (2001) 713-719.

[14] H. Berger, Über das elektroenkephalogramm des menschen, Arch. Psychiatr. Nervenkr. 87 (1929) 527-570.

[15] S. Baillet, J.C. Mosher, R.M. Leahy, Electromagnetic brain mapping, IEEE Signal Proc. Mag. November (2001) 14-30.

[16] E.R. Kandel, J.H. Schwartz, T.M. Jessell, Principles of Neural Science, 4th ed. McGraw Hill, NY, 2000.

[17] P.L. Nunez, Neocortical Dynamics and Human EEG Rhythms, Oxford University Press, USA, 1995.

[18] P.L. Nunez, Toward a quantitative description of large-scale neocortical dynamic function and EEG, Behav. Brain Sci. 23 (2000) 371-437.

[19] A.S. Gevins, J.C. Doyle, B.A. Cutillo, R.E. Schaffer, R.S. Tannenhill, J.H. Ghannam, V.A. Gilcrease, C.L. Yeager, Electrical potentials in human brain during cognition: new methods reveal dynamic patterns of correlation, Science 213 (August) (1981) 918-922.

[20] A.S. Gevins, R.E. Schaffer, J.C. Doyle, B.A. Cutillo, R.S. Tannehill, S.L. Bressler, Shadows of thought: shifting lateralization of human brain electrical patterns during brief visuomotor tasks, Science 220 (1983) 97-99.

[21] E. Rodriguez, N. George, J.-P. Lachaux, J. Martinerie, B. Renault, F.J. Varela Perception's shadow: long-distance synchronization of human brain activity, Nature 397 (February) (1999) 430-433.

[22] L.C. Parra, C.D. Spence, A.D. Gerson, P. Sajda, Recipes for linear analysis of EEG NeuroImage 28 (2005) 326-341.

[23] J. Vidal, Real-time detection of brain events in EEG, Proc. IEEE 65 (May (5)) (1977) 633-641.

[24] C. Phillips, J. Mattout, M.D. Rugg, P. Maquet, K.J. Friston, An empirical Bayesian solution to the source reconstruction problem in EEG, NeuroImage 24 (2005) 997-1011.

[25] D. Garrett, D.A. Peterson, C.W. Anderson, M.H. Thaut, Comparison of linear, nonlinear, and feature selection methods for EEG signal classification, IEEE Trans. Neural Syst. Rehabil. Eng. 11 (June (2)) (2003) 141-144.

[26] Z.A. Keirn, J.I. Aunon, A new mode of communication between man and his surroundings, IEEE Trans. Biomed. Eng. 37 (December (12)) (1990) 1209-1214.

[27] P.L. Nunez, R.B. Silberstein, On the relationship of synaptic activity to macroscopic measurements: does co-registration of EEG with fMRI make sense? Brain Topogr. 13 (2000) 79-96.

[28] P.L. Nunez, Electric Fields of the Brain, New York, Oxford, 1981.

[29] EEGLAB - Open Source MATLAB Tool Box for Eletrophysiological Research, 2007, sccn.ucsd.edu/eeglab/.

[30] T. Lan, D. Erdogmus, A. Adami, S. Mathan, M. Pavel, Channel selection and feature projection for cognitive load estimation using ambulatory EEG, Comp. Intell. Neurosci. (2007), article ID 74895, http://www. pubmedcentral.nih.gov/picrender.fcgi?artid=2267884\&blobtype=pdf (online).

[31] K.V.R. Ravi, R. Palaniappan, A minimal channel set for individual identification with EEG biometric using genetic algorithm, in: Int. Conf. Comp. Intell. Multimedia Appln., 13-15 December, 2007, pp. 328-332.

[32] K. Majumdar, Constraining minimum-norm inverse by phase synchronization and signal power of the scalp EEG channels, IEEE Trans. Biomed. Eng. 56 (April (4)) (2009) 1228-1235.

[33] S. Roweis, S. Saul, Nonlinear dimensionality reduction by locally linear embedding, Science 290 (2000) 2323-2326.

[34] C. Robert, J.-F. Gaudy, A. Limoge, Electroencephalogram processing using neural networks, Clin. Neurophysiol. 113 (2000) 694-701.

[35] A.K. Tun, N.T. Lye, U.R. Abeyrante, P. Saratchandran, RBF networks for source localization in quantitative electrophysiology, Crit. Rev. Biomed. Eng. 28 (3-4) (2000) 463-472.

[36] S.R. Benbadis, D. Rielo, EEG Artifacts, eMedicine, 2008, Available from: http://emedicine.medscape.com/article/1140247-overview, September.

[37] P.K. Sadasivan, D.N. Dutt, Minimization of EOG artifacts from corrupted EEC signals using a neural network approach, Comput. Biol. Med. 24 (6) (1994) 441-449. 
[38] F.Y. Wu, J.D. Slater, R.E. Ramsey, Neural network approach in multichannel auditory event-related potential analysis, Int. J. Biomed. Comput. 35 (1994) $157-168$.

[39] J. Wu, E.C. Ifeachor, E.M. Allen, N.R. Hudson, A neural network based artifact detection system for EEG signal processing, in: Proc. Int. Conf. Neural Networks Expert Syst. Med. Healthcare, University of Plymouth, 1994, pp. 257-266.

[40] P. Durka, R. Kiezyk, K. Blinowska, Neural Networks and Wavelet Analysis in EEG Artifact Recognition, 1996, Available from: http://brain.fuw. edu.pl/ durka/papers/medicon98_arif.pdf.

[41] E.P. Riddington, J. Wu, E.C. Ifeachor, E.M. Allen, N.R. Hudson, Intelligent enhancement and interpretation of EEG signals, in: Proc. IEE Colloquium on Artif. Intell. Methods for Biomed. Data Processing, Savoy Place, London, 1996, pp. $11 / 1-11 / 7$.

[42] C. Herrmann, Symbolical reasoning about numerical data: a hybrid approach, Appl. Intell. 7 (1997) 339-354.

[43] A. Saastamoinen, T. Pietila, A. Varri, M. Lehtokangas, J. Saarinen, Waveform detection with RBF network - application to automated EEG analysis, Neurocomputing 20 (1998) 1-13.

[44] R. Bogacz, U. Markowska-Kaczma, A. Kozik, Blinking artifact recognition in EEG signal using artificial neural networks, in: Proc. 4th Conf. Neural Networks \& Applications, Zakopane, Poland, 1999, p. 6.

[45] R.C. Vasko, J. Carrier, P.D. Nowell, J.P. Monahan, J.D. Cashmere, D.J. Buysse, M.E. Thase, D.J. Kupfer, A neural network for rejecting artifacts from eyesopen awake EEG, in: Proc. 14th Annual APSS Meeting, Las Vegas, Nevada, 17-20 June, 2000.

[46] V. Schetinin, Polynomial neural networks learnt to classify EEG signals, in: NIMIA-SC2001, Crema, Italy, 9-20 October, 2001, Available from: http://arxiv.org/ftp/cs/papers/0504/0504058.pdf.

[47] Z.J. Koles, Trends in EEG source localization, Eletroencephalogr. Clin. Neurophysiol. 106 (February) (1998) 127-137.

[48] H. Hallez, B. Vanrumste, R. Grech, J. Muscat, W. De Clercq, A. Vergult, Y. D'Asseler, K.P. Camilleri, S.G. Fabri, S. Van Huffel, I. Lemahieu, Review on solving the forward problem in EEG source analysis, J. Neuroeng. Rehabil. 4 (2007), Available from: http://www.jneuroengrehab.com/content/4/1/46 (open access).

[49] R. Grech, T. Cassar, J. Muscat, K.P. Camilleri, S.G. Fabri, M. Zervakis, P. Xanthopoulos, V. Sakkalis, B. Vanrumste, Review on solving the inverse problem in EEG source analysis, J. Neuroeng. Rehabil. 5 (2008), Available from: http://www.jneuroengrehab.com/content/5/1/25 (open access).

[50] U.R. Abeyratne, Y. Kinouchi, H. Oki, J. Okada, F. Shichijo, K. Matsumoto, Artificial neural networks for source localization in human brain, Brain Topogr. 4 (August (1)) (1991) 3-21.

[51] G. Van Hoey, J. De Clercq, B. Vanrumste, R. Van de Walle, I. Lemahieu, M. DHave, P. Boon, EEG dipole source localization using artificial neural networks, Phys. Med. Biol. 45 (2000) 997-1011.

[52] R. Abeyratne, G. Zhang, P. Saratchandran, EEG source localization: a comparative study of classical and neural networks methods, Int. J. Neural Syst. 11 (4) (2001) 349-360.

[53] R.J. Sclabassi, M. Sonmez, M. Sun, EEG source localization: a neural network approach, Neurol. Res. 23 (5) (2001) 457-464.

[54] M. Sun, R.J. Sclabassi, The forward EEG solution can be computed using artificial neural networks, IEEE Trans. Biomed. Eng. 47 (8) (2000) 1044-1050.

[55] A.K. Tun, N.T. Lye, Z. Guanglan, U.R. Abeyratne, P. Saratchandran, RBF networks for source localization in quantitative electrophysiology, Crit. Rev. Biomed. Eng. 28 (2000) 463-472.

[56] M. Yuasa, Q. Zhang, H. Nagashino, Y. Kinouchi, EEG source localization for two dipoles by neural networks, in: Proceedings of the 20th IEEE Annual International Conference IEEE/EMBS, 29 October-1 November, Hong Kong, 1998, pp. 2190-2192.

[57] Q. Zhang, M. Yuasa, H. Nagashino, Y. Kinoushi, Single dipole source localization from conventional EEG using BP neural networks, in: Proceedings of the 20th IEEE Annual International Conference IEEE/EMBS, 29 October-1 November, Hong Kong, 1998, pp. 2163-2166.

[58] Z. Li, M. Akutagawa, Y. Kinouchi, Brain source localization for two dipoles using a combined method from 32-channel EEGs, in: Proc. IEEE Engineering in Medicine and Biology Soc. Conf., 2005, pp. 4518-4521.

[59] S.S. Cash, E. Halgren, N. Dehghani, A.O. Rossetti, T. Thesen, C. Wang, O. Devinsky, R. Kuzniecky, W. Doyle, J.R. Madsen, E. Bromfield, L. Eross, P. Halasz, G. Karmos, R. Csercsa, L. Wittner, I. Ulbert, Human K-complex represents an isolated cortical down-state, Science 324 (2009) 1084-1087.

[60] I.S. Bankman, V.G. Sigillito, R.A. Wise, P.L. Smith, Feature-based detection of the K-complex wave in the human electroencephalogram using neural networks, IEEE Trans. Biomed. Eng. 39 (12) (1992) 1305-1310.

[61] E. Huupponen, A. Varri, S.L. Himanen, J. Hasan, M. Lehtokangas, J. Saarinen, Autoassociative MLP in sleep spindle detection, J. Med. Syst. 24 (3) (2000) 183-193.

[62] D.C. Reddy, D.R. Korrai, Neural networks for classification of EEG signals, Medinfo 92 (1992) 653-658.

[63] J.C. Principe, A.M.P. Tome, Performance and training strategies in feed forward neural networks: an application to sleep scoring, Proc. IJCNN 89 (1989) 341-346.

[64] G. Pfurtscheller, D. Flotzinger, K. Matuschik, Sleep classification in infants based on artificial neural networks, Biomed. Technol. 37 (1992) $122-130$.
[65] A.S. Gevins, N.H. Morgan, Applications of neural-network (NN) signal processing in brain research, IEEE Trans. Acoust. Speech Signal Process. 36 (July (7)) (1988) 1152-1162.

[66] C. Robert, C. Guilpin, A. Limoge, A review of neural network application in sleep research, J. Neurosci. Methods 79 (1998) 187-193.

[67] N. Schaltenbrand, R. Lengelle, M. Toussaint, R. Luthringer, G. Carelli, A. Jacqumin, E. Lainey, A. Muzet, J.P. Macher, Sleep stage scoring using neural network model: comparison between visual and automatic analysis in normal subjects and patients, Sleep 20 (1) (1996) 26-35.

[68] D. Liu, Z. Pang, S.R. Lloyd, A neural network method for detection of obstructive sleep apnea and narcolepsy based on pupil size and EEG, IEEE Trans. Neural Netw. 19 (February (2)) (2008) 308-318.

[69] R. Lin, R.-G. Lee, C.-L. Tseng, H.-K. Zhou, C.-F. Chao, J.-A. Jiang, A new approach for identifying sleep apnea syndrome using wavelet transform and neural networks, Biomed. Eng. Appl. Basis Commun. 18 (June (3)) (2006) 138-143.

[70] R.K. Sinha, Artificial neural network and wavelet based automated detection of sleep spindles, REM sleep and wake states, J. Med. Syst. 32 (2008) 291-299.

[71] A. Vuckovic, V. Radivojevic, A.C.N. Chen, D. Popovic, Automatic recognition of alertness and drowsiness from EEG by an artificial neural network, Med. Eng. Phys. 24 (2002) 349-360.

[72] K.H. Finley, J.B. Dynes, Electroencephalographic studies in epilepsy: a critical analysis, Brain 65 (1942) 256-265.

[73] J. Gotman, Automatic recognition of epileptic seizure in the EEG, Electroencephalogr. Clin. Neurophysiol. 54 (1982) 530-540.

[74] P. Elo, J. Saarinen, A. Varri, H. Nieminen, K. Kaski, Classification of epileptic EEG by using self-organizing maps, in: I. Aleksander, J. Taylor (Eds.), Artificial neural networks, vol. 2, Elsevier, 1992, pp. 1147-1150.

[75] A.J. Gabor, M. Seyal, Automated interictal EEG spike detection using artificial neural networks, Electroencephalogr. Clin. Neurophysiol. 83 (1992) 271-280.

[76] G. Jando, R.M. Siegel, Z. Horvath, G. Buzsaki, Pattern recognition of the electroencephalogram by artificial neural networks, Electroencephalogr. Clin. Neurophysiol. 86 (1993) 100-109.

[77] W.R.S. Webber, B. Litt, K. Wilson, R.P. Lesser, Practical detection of epileptiform discharges (Eds) in the EEG using an artificial neural network: a comparison of raw and parameterized EEG data, Electroencephalogr. Clin. Neurophysiol. 91 (1994) 194-204.

[78] N. Pradhan, P.K. Sadasivan, G.R. Arunodaya, Detection of seizure activity in EEG by an artificial neural network: a preliminary study, Comput. Biomed. Res. 29 (1996) 303-313.

[79] M.Varsta, J.Heikkonen, J. del, R. Millan, Epileptic activity detection in EEG with neural networks, in: Proc. 3rd Int. Conf. Eng. Applications of Neural Networks, Stockholm, Sweden, 16-18 June, 1997, pp. 179-186.

[80] A.J. Gabor, Seizure detection using a self-organizing neural network: validation and comparison with other detection strategies, Electroencephalogr. Clin. Neurophysiol. 107 (1998) 27-32.

[81] S. Walczack, W.J. Nowack, An artificial neural network approach to diagnosing epilepsy using lateralized bursts of theta EEGs, J. Med. Syst. 25(1)(2001) 9-20.

82] V.P. Nigam, D. Graupe, A neural-network-based detection of epilepsy, Neurol. Res. 26 (2004) 55-60.

[83] A. Subasi, E. Ercelebi, Classification of EEG signals using neural networks and logistic regression, Comput. Methods Programs Biomed. 78 (2005) 87-99.

[84] A. Subasi, Application of adaptive neuro-fuzzy inference system for epileptic seizure detection using wavelet feature extraction, Comput. Biol. Med. 37 (2007) 227-244

[85] A. Aarabi, F. Wallois, R. Grebe, Automated neonatal seizure detection: a multistage classification system through feature selection based on relevance and redundancy analysis, Electroencephalogr. Clin. Neurophysiol. 117 (February (2)) (2006) 328-340.

[86] A. Aarabi, R. Grebe, F. Wallois, A multistage knowledge-based system for EEG seizure detection in newborn infants, Electroencephalogr. Clin. Neurophysiol. 118 (December (12)) (2007) 2781-2797.

[87] N.B. Karayiannis, A. Mukherjee, J.R. Glover, P.Y. Kotnas, J.D. Frost Jr., R.A. Hrachovy, E.M. Mizrahi, Detection of pseudosinusoidal epileptic seizure segment in the neonatal EEG by cascading a rule-based algorithm with a neural network, IEEE Trans. Biomed. Eng. 53 (April (4)) (2006) 633-641.

[88] V. Srinivasan, C. Eswaran, N. Sriraam, Artificial neural network based epileptic detection using time-domain and frequency-domain features, J. Med. Syst. 29 (December (6)) (2005) 647-660.

[89] A.Petrosian, D. Prokhorov, R. Homan, R. Dasheiff, D.I. Wunch, Recurrent neural network based prediction of epileptic seizure in intra- and extracranial EEG, Neurocomputing 30 (2000) 201-218.

[90] C.-W. Ko, H.-W. Chung, Automatic spike detection via an artificial neural network using raw EEG data: effects of data preparation and implications in the limitations of online recognition, Clin. Neurophysiol. 111 (3)(2000) 477-481.

[91] N.F. Guler, E.D. Ubeyli, I. Guler, Recurrent neural networks employing Lyapunov exponents for EEG signal classification, Expert Syst. Appl. 29 (October (3)) (2005) 506-514

[92] C. Sahin, S.N. Ogulata, K. Aslan, H. Bozdemir, The applications of neural networks in classification of epilepsy using EEG signals, in: LNCS: Advances in Brain. Vision and Artificial Intelligence, Springer, Heidelberg, 2007, pp. 499-508.

[93] A.S. Gevins, Analysis of electromagnetic signals of the human brain: milestones, obstacles, and goals, IEEE Trans. Biomed. Eng. 31 (December (12)) (1984) 833-850. 
[94] L.A. Farwell, E. Donchin, Talking off the top of your head: toward a mental prosthesis utilizing event-related brain potentials, Electroencephalogr. Clin. Neurophysiol. 70 (1988) 510-523.

[95] G. Pfurtscheller, D. Flotzinger, W. Mohl, M. Peltoranta, Prediction of the side of hand movements from single trial multi-channel EEG data using neural networks, Electroencephalogr. Clin. Neurophysiol. 82 (1992) 313-315.

[96] N. Masic, G. Pfurtscheller, Neural network based classification of single-trial EEG data, Artif. Intell. Med. 5 (1993) 503-513.

[97] M. Peltoranta, G. Pfurtscheller, Neural network based classification of nonaveraged event-related EEG responses, Med. Biol. Eng. Comput. 32 (2)(1994) 189-196.

[98] N. Masic, G. Pfurtscheller, D. Flotzinger, Neural network-based predictions of hand movements using real and simulated EEG data, NeuroComputing 7 (April (3)) (1995) 259-274.

[99] B.O. Peters, G. Pfurtscheller, H. Flyvbjerg, Automatic differentiation of multichannel EEG signals, IEEE Trans. Biomed. Eng. 48 (January (1)) (2001) 111-116.

[100] B.O. Peters, G. Pfurtscheller, H. Flyvbjerg, Mining multi-channel EEG for its information content: an ANN-based method for a brain-computer interface, Neural Netw. 11 (1998) 1429-1433.

101] K.S. Fung, F.H. Chan, F.K. Lam, P.W. poon, A tracing evoked potential estimator, Med. Biol. Eng. Comput. 37 (March (2)) (1999) 218-227.

[102] F. Babiloni, F. Carducci, S. Cerutti, D. Liberati, P.M. Rossini, A. Urbano, C. Babiloni, Comparison between human and artificial neural network detection of Laplacian-derived electroencephalographic activity related to unilateral voluntary movements, Comput. Biomed. Res. 33 (2000) 59-74.

[103] J. del, R. Millan, J. Mourino, M.G. Marciani, F. Babiloni, F. Topani, I. Canale, J Heikkonen, K. Kaski, Adaptive brain interfaces for physically-disabled people, in: 20th Ann. Int. Conf. IEEE Eng. Med. \& Biol. Soc., vol. 4, Hong Kong, October 29-November 1, 1998, pp. 2008-2011.

[104] W.D. Penny, S.J. Roberts, EEG-based communication via dynamic neural network models, Proc. Int. Joint Conf. Neural Netw. 5 (1999) 3586-3590.

[105] K. Nakayama, Y. Kaneda, A. Hirano, A brain computer interface based on FFT and multilayer neural network feature extraction and generalization, in: Int. Sym. Intell. Signal Proc. Commun. Syst., November 28-December 1, 2007, pp. 826-829.

[106] X. Liu, S. Hibino, T. Hanai, T. Imanishi, T. Shirataki, T. Ogawa, H. Honda, T. Kobayashi, Construction of an electroencephalogram-based brain-computer interface using an artificial neural network, IEICE Trans. Inf. Syst. E86-D (9) (2003) 1879-1886.

[107] C.-J. Lin, M.-H. Hsieh, Classification of mental task from EEG data using neural networks based on particle swarm optimization, NeuroComputing January (72) (2009) 1121-1130

[108] D.H. Lange, H.T. Siegelmann, H. Pratt, G.F. Inbar, Overcoming selective ensemble averaging: unsupervised identification of event-related brain potentials, IEEE Trans. Biomed. Eng. 47 (June (6)) (2000) 822-826

[109] N. Gong, J. Duchene, Visual evoked potential classification for clinical use, in Proc. 17th Annu. Conf. IEEE Eng. Biol. Med. Soc., vol. 2, 20-23 September, 1995, pp. 917-918.

[110] S.-M. Fung, Fast evoked potential estimation by artificial neural networks, PhD Thesis, University of Hong Kong, 1999, Available from: http://hub.hku.hk/handle/123456789/31753.

[111] A. Uncini, M. Marchesi, G. Orlandi, F. Piazza, An adaptive neural network filter for evoked potential, in: Proc. of ISCAS-90 IEEE Int. Symposium on Circuit and Systems, New Orleans, USA, May, 1990, pp. 1086-1089.

[112] A. Dumitras, A.T. Murgan, V. Lazarescu, A quantitative study of evoked potential estimation using feed forward neural network, in: Proc. IEEE Workshop on Neural Networks for Signal Processing, 6-8 September, 1994, pp. 606-615.

[113] K.S.M. Fung, F.K. Lam, F.H.Y. Chan, P.W.F. Poon, J.G. Lin, Adaptive neural network filter for visual evoked potential estimation, Proc. IEEE Int. Conf. Neural Netw. 5 (November/December) (1995) 2293-2296.

[114] N. Laskaris, S. Fotopoulos, P. Papathanasopoulos, A. Bezerianos, Robust moving averages with Hopfield neural network network implementation for monitoring evoked potential signals, Eletroencephalogr. Clin. Neurophysiol. 104 (1997) 151-156.

[115] L. Leistritz, K. Hoffmann, M. Galicki, H. Witte, Identification of hemifield single trail PVEP on the basi of generalized dynamic neural network classifiers, Clin. Neurophysiol. 110 (1999) 1978-1986.

[116] T.J. Dasey, E. Micheli-Tzanakou, An unsupervised neural network system for visual evoked potential, in: E. Micheli-Tzanakou (Ed.), Supervised and Unsupervised Pattern Recognition: Feature Extraction and Computational Intelligence, CRC Press, Boca Raton, FL, USA, 2000, pp. 186-194.

[117] K. Hoffmann, L. Leistritz, M. Feucht, F. Benninger, D. Reiter, H. Witte, Identification of the stimulated hemiretina in primary school children and adults based on left and right hemifield pattern reversal visual evoked potentials a comparative study, Clin. Neurophysiol. 112 (2) (2001) 359-368.

[118] W. Qiu, K.S.M. Fung, F.H.Y. Chan, F.K. Lam, P.W.F. Poon, R.P. Hamernik, Adaptive filtering of evoked potentials with radial-basis-function neural network prefilter, IEEE Trans. Biomed. Eng. 49 (March (3)) (2002) 225-232.

[119] C.J. Du, H.E. Yin, S.C. Wu, X.Y. Ren, Y.J. Zeng, Y.F. Pan, Visual evoked potentials estimation by adaptive noise cancellation with neural-network-based fuzzy inference system, in: 26th Annual Int. Conf. IEEE Eng. Med. Biol. Soc. vol. 1 1-5 September, 2004, pp. 624-627.

[120] R. Palaniappan, Method of identifying individuals using VEP signals and neura network, IEE Proc. Sci. Meas. Technol. 151 (January (1)) (2004) 16-20.
[121] R. Sivakumar, G. Ravindran, Automatic discrimination of abnormal subjects using the visual evoked potential spectral components, J Biomed. Biotechnol. 2004 (April (1)) (2004) 5-9, Available from: http:// www.pubmedcentral.nih.gov/picrender.fcgi?artid=545650\&blobtype=pdf.

[122] A. Mghari, M. Himmi, A. Amaloud, F. Regragui, Visual evoked potentials discrimination based on adaptive zero-tracking neural network, Comput. Biol. Med. 36 (4) (2006) 408-418.

[123] I. Bruha, G.P. Madhavan, Combined syntax - neural net method for pattern recognition of evoked potentials, in: R. Janicki, W.W. Koczkodaj (Eds.), Computation and Information, Elsevier, 1989, pp. 361-369.

[124] I. Bruha, G.P. Madhavan, M.S.-K. Chong, Use of multilayer perceptron for recognition of evoked potential, Int. J. Pattern Recogn. Artif. Intell. 4 (December (4)) (Dec 1990) 705-716.

[125] D.T. Freeman, Computer recognition of brain stem auditory evoked potential wave V by a neural network, Proc. Annu. Symp. Comput. Appl. Med. Care (1991) 305-309.

[126] J.B.A. Habraken, M.J. van Gils, P.J.M. Cluitmans, Identification of peak V in brainstem auditory evoked potentials with neural networks, Comput. Biol. Med. 23 (5) (1993) 369-380.

[127] D. Alpsan, M. Towsey, O. Ozdamar, D.N. Ghista, Determining the hearing threshold from brain stem evoked potential: optimizing a neural network to improve classification performance, IEEE Eng. Med. Biol. Mag. 13 (August/September (4)) (1994) 465-471.

[128] A. Nayak, M.S. Roy, R.J. Roy, Neural networks for predicting depth of anesthesia from auditory evoked potentials: a comparison of wavelet transform with autoregressive modeling and power spectrum feature extraction methods, in: 17th Annu. Conf. IEEE Eng. Med. Biol. Soc., vol. 1, 20-25 September, 1995 pp. 797-798.

[129] Y.-Y. Lu, J.W. Huang, R.J. Roy, Estimation of depth of anesthesia using the midlatency auditory evoked potentials by means of neural network based multiple classifier system, in: Proc. 19th Int. Annu. Conf. IEEE Eng. Med. Biol. Soc., vol. 3, 30 October-2 November, 1997, pp. 1100-1103.

[130] J.K. Backory, D.A. Linkens, J.E. Peacock, Neural network based prediction of depth of anesthesia using auditory evoked potentials with a wavelet transform, Biomed. Eng. Appl. Basis Commun. 10 (4) (1998) 217-224.

[131] U. Hoppe, S. Weiss, R.W. Stewart, U. Eysholdt, An automatic sequential recognition method for cortical auditory evoked potentials, IEEE Trans. Biomed. Eng. 48 (February (2)) (2001) 154-164.

[132] X.-S. Zhang, R.J. Roy, D. Schwender, M. Daunderer, Discrimination of anesthetic states using mid-latency auditory evoked potential and artificial neural networks, Biomed. Eng. 29 (May (5)) (2001) 446-453.

[133] E. Thomas, P.S. Sandor, A. Amborsini, J. Schoenen, A neural network model of sensitization of evoked cortical responses in migraine, Cephalalgia 22 (March (1)) (2002) 48-53.

[134] A. Guiterrez, A. Somolinos, Preprocessing of brain stem auditory evoked potentials for diagnosing multiple sclerosis, in: Proc. 2nd IASTED Int. Conf Adv. Comp. Sc. Tech., Puerto Vallarta, Mexico, 2006, pp. 196-201.

[135] D. Liberati, A neural network for single sweep brain evoked potential detection and recognition, in: Proc. IV Int. Symp. Biomed. Eng., Peniscola, Spain, 1991, pp. 484-485.

[136] D.B. Smith, D.F. Lovely, A neural network based approach to whitening biological noise for somatosensory evoked potential detection, in: Proc. 17th Annu. Conf. IEEE Eng. Med. Biol. Soc., vol. 1, 20-25 September, 1994, pp. 803-804.

[137] R.C.W. Grieve, P.A. Parker, B. Hudgins, Training neural networks for stimulus artifact reduction in somatosensory evoked potential, in: Proc. 18th Annu. Int. Conf. IEEE Eng. Med. Biol Soc., vol. 3, 31 October-3 November, 1996, pp. 917-918.

[138] A.C. Merzagora, F. Bracchi, S. Cerutti, L. Rossi, A.M. Bianchi, A. Gaggiani, A radial basis function neural network for single sweep detection of somatosensory evoked potential, in: Proc. 26th Annu. Int. Conf. IEEE EMBS, San Francisco, CA USA, 1-5 September, 2004, pp. 427-430.

[139] B.H. Boudreau, K.B. Englehart, A.D. Chan, P.A. Parker, Reduction of stimulus artifact in somatosensory evoked potentials: segmented versus subthreshold training, IEEE Trans. Biomed. Eng. 51 (July (7)) (2004) 1187-1195.

[140] L. Gupta, D.L. Molfese, R. Tammana, An artificial neural network approach to ERP classification, Brain Cognit. 27 (April (3)) (1995) 311-330.

[141] G. Belenky, H.C. Sing, M.L. Thomas, Y. Shaham, S. Balwinski, D.P. Redmond T.J. Balkin, Discrimination of rested from sleep-deprived EEG in awake normal humans by artificial neural network, in: Proc. IEEE Int. Conf. Neural Networks, Piscataway, NJ, USA, 1994, pp. 3521-3524.

[142] A. Gulati, D. Jacobs, M.N. Silan, A.T. Pope, Classification and prediction of electroencephalographic data with hybrid neural networks to analyze human-automation related-effects, in: Proc. 7th Int. Conf. Art. Intell. Expert Syst. Appl., November, 1995, pp. 449-454.

[143] T.-P. Jung, S. Makeig, M. Stensmo, T.J. Sejnowski, Estimating alertness from the EEG power spectrum, IEEE Trans. Biomed. Eng. 44 (January (1)) (1997) 60-69.

[144] V. Tuulik, A. Raja, A. Meister, E. Lossmann, Neural network method to determine the vigilance levels of the central nervous system related to occupational chronic chemical stress, Technol. Health Care 5 (1997) 243-251.

[145] C. Jouny, Electrophysiolgie des etats de vigilance chez l'homme, PhD thesis, University of Strasbourg, 1997.

[146] A.S. Gevins, M.E. Smith, Detecting transient cognitive impairment with EEG pattern recognition methods, Clin. Neurophysiol. 70 (10) (1999) 1018-1024. 
[147] A. Subasi, K. Kiymik, M. Akin, O. Erogul, Automatic recognition of vigilance state by using wavelet-based artificial neural network, Neural Comput. Appl. 14 (March (1)) (2005) 45-55.

[148] K. Holthausen, O. Breidbach, B. Scheidt, J. Frenzel, Brain dysmaturity index for automatic detection of high-risk infants, Pediatr. Neurol. 22 (3) (2000) 187-191.

[149] L. Moreno, J.D. Pineiro, J.L. Sanchez, S. Manas, J. Merino, L. Acosta, A. Hamilton, Brain maturation estimation using neural classifier, IEEE Trans. Biomed. Eng. 42 (April (4)) (1995) 428-432.

[150] R. Sanchez, A. Riquenes, M. Perez-Abalo, Automatic detection of auditory brainstem responses using feature vectors, Int. J. Bio-Med. Comput. 39 (June (3)) (1995) 287-297

[151] H. Heinrich, H. Dickhaus, A. Rothenberger, V. Heinrich, G.H. Moll, Single-sweep analysis of event-related potentials by wavelet networks methodological basis and clinical applications, IEEE Trans. Biomed. Eng. 46 (July (7)) (1999) 867-879.

[152] H. Heinrich, G.H. Moll, H. Dickhaus, V. Kolev, J. Yordanova, A. Rothenberger, Time-on-task analysis using wavelet networks in an event-related potential study on attention-deficit hyperactivity disorder, Clin. Neurophysiol. 112 (7) (2001) 1280-1287.

[153] M. de Tommaso, V. Sciruicchio, R. Bellootti, M. Castellano, P. Tota, M. Guido, G. Sasanelli, F.M. Puca, Discrimination between migraine patients normal subjects based on steady state visual evoked potentials: discriminant analysis and artificial neural network classifier, Funct. Neurol. 12 (6) (1997) 333-338.

[154] M. de Tommaso, V. Sciruicchio, R. Bellootti, M. Guido, G. Sasanelli, L.M. Specchio, F. Puca, Photic driving response in primary headache: diagnostic value testes by discriminant analysis and neural network classifiers, Ital. J. Neurol. Sci. 20 (1999) 23-28.

[155] R. Bellotti, F. De Carlo, M. de Tommaso, M. Lucente, Migraine detection through spontaneous EEG analysis, in: Proc. 29th Annu. Int. Conf. IEEE Eng. Med. Biol. Soc., 22-26 August, 2007, pp. 1834-1837.

[156] S. Kara, A. Guven, Neural network-based diagnosis for optic nerve disease from visual-evoked potential, J. Med. Syst. 31 (July (5)) (2007) 391-396.

[157] F.Y. Wu, J.D. Slater, L.S. Honig, R. Eugene Ramsey, A neural network design for event-related potential diagnosis, Comput. Biol. Med. 23 (May (3)) (1993) 251-264.

[158] J. Magdolen, P. Rappelsberger, G. Dorffner, A. Flexer, G. Winterer, Application of non-linear classifiers to EEG at rest for identifying psychiatric disorders, in: Proc. 13th Eur. Meeting on Cybernetics and Systems Research (EMCR'96), 2 vols., Austrian Society for Cybernetic Studies, Vienna, 1996, pp. 592-596.

[159] G. Papadourakis, M. Vourkas, S. Micheloyannis, B. Jervis, Use of artificial neural networks for clinical diagnosis, Math. Comput. Simul. 40 (1996) 623-635.

[160] J.R. Sveinsson, J.A. Benediktsson, S.B. Stefansson, K. Davidsson, Parallel principal component neural networks for classification of event-related potential waveforms, Med. Eng. Phys. 19 (1) (1997) 15-20.

[161] Y.-J. Li, F.-Y. Fan, Classification of schizophrenia and depression by EEG with ANNs, in: Proc. 27th Int. Conf. IEEE Eng. Med. Biol. Soc., 17-18 January, 2005, pp. 2679-2682.

[162] G. Papadourakis, M. Vourkas, S. Micheloyannis, B. Jervis, Use of artificial networks for clinical diagnosis, Math. Comput. Simul. 40 (May) (1996) 623-635.

[163] B.W. Jervis, M.R. Saatchi, S. Yu, R.F. Harrison, C.P. Lim, S. Oke, Application of artificial neural networks to CNV data to investigate, Psychiatry Res.: Neuroimaging 68 (February (2)) (1997) 166-1166.

[164] R. Polikar, M.H. Greer, L. Udpa, F. Keinert, Multiresolution wavelet analysis of ERPs for the detection of Alzheimer's disease, in: Proc. 19th Annu. Int. Conf. IEEE Eng. Med. Biol. Soc., vol. 3, 30 October-2 November, 1997, pp. 1301-1304.

[165] S. Hibino, T. Hanai, E. Nagata, M. Matsubara, K. Fukagawa, T. Shirataki, H. Honda, T. Kobayashi, An assessment system of dementia of Alzheimer type using artificial neural networks, in: H. Malmgren, M. Borga, L. Niklasson (Eds.), Artificial Neural Networks in Medicine and Biology, Springer-Verlag, 2000, pp. 180-185.

[166] A.A. Petrosian, D.V. Prokhodov, W. Lajara-Nanson, R.B. Schiffer, Recurrent neural network-based approach for early recognition of Alzheimer's disease in EEG, Clin. Neurophysiol. 112 (2001) 1378-1387.

[167] H.T. Kim, B.Y. Kim, E.H. Park, J.W. Kim, E.W. Hwang, S.K. Han, S. Cho, Computerized recognition of Alzheimer disease-EEG using genetic algorithms and neural network, Future Gen. Comput. Syst. 21 (July (7)) (2005) 1124-1130.

[168] B.W. Jervis, M.R. Saatchi, A. Lacey, G.M. Papadourakis, M. Vourkas, T. Roberts, E.M. Allen, N.R. Hudson, S. Oke, The application of unsupervised artificial neural networks to the subclassification of subjects at-risk of Huntington's disease, in: Proc. IEE Coll. Int. Dec. Sup. Syst. Med., 15 June, 1992, pp. 5/1-5/9.

[169] B.W. Jervis, T. Garcia, E.P. Giahnakis, Probabilistic simplified fuzzy ARTMAP (PSFAM), IEE Proc. Sci. Meas. Technol. 146 (4) (1999) 165-169.

[170] M. de Tommaso, F. De Carlo, O. Difruscolo, R. Massafar, V. Sciruicchio, R. Bellotti, Detection of subclinical brain electrical activity changes in Huntington's disease using artificial neural networks, Clin. Neurophysiol. 114 (7) (2003) 1237-1245.

[171] R. Bellotti, F. De Carlo, R. Massafra, M. de Tommaso, V. Sciruicchio, Topographic classification of EEG patterns un Huntington's disease, Neurol. Clin. Neurophysiol. 37 (November) (2004), Available from: http:// www.neurojournal.com/article/view/182/126.

[172] B. Kloppel, Classification by neural networks of evoked potentials, Neuropsychobiology 29 (1994) 47-52.

[173] R. Palaniappan, Screening for chronic alcoholic subjects using multiple gamma band EEG: a pilot study, JCS\&T 7 (April (2)) (2007) 182-185.
[174] I. Guler, E.D. Ubeyli, Multiclass support vector machines for EEG-signal classification, IEEE Trans. Inform. Technol. Biomed. 11 (March (2)) (2007) 117-126.

[175] E.D. Ubeyli, Implementing eigenvector methods/probabilistic neural networks for analysis of EEG signals, Neural Netw. 21 (2008) 1410-1417.

[176] R. Begg, J. Kamaruzzaman, R. Sarker, Neural Networks in Healthcare: Potential and Challenges, IGI Publishing, USA, 2006.

[177] V. Lalitha, C. Eswaran, Automated detection of anesthetic depth levels using chaotic features with artificial neural networks, J. Med. Syst. 31 (December (6)) (2007) 445-452.

[178] C. Robert, P. Karasinski, C.D. Arreto, J.F. Gaudy, Monitoring anesthesia using neural networks: a survey, J. Clin. Monit. Comput. 17 (April (3-4)) (2002) 259-267.

[179] R. Palaniappan, D.P. Mandic, Biometrics from brain electrical activity: a machine learning approach, IEEE Trans. Pattern Anal. Mach. Intell. 29 (April (4)) (2007) 738-742.

[180] R.A. Veselis, R. Reinsel, S. Sommer, G. Carlon, Use of neural network analysis to classify electroencephalographic patterns against depth of midazolam sedation in intensive care, J. Clin. Monit. Comput. 7 (July (3)) (1991) 259-267.

[181] A.S. Gevins, R.K. Stone, S.D. Ragsdale, Differentiating the effects of three benzodiazepines on non-REM sleep EEG spectra, Neuropsychobiology 19 (1988) $108-115$.

[182] R.A. Veselis, R. Reinsel, M. Wronski, Analytical methods to differentiate similar electroencephalographic spectra: neural network and discriminant analysis, J. Clin. Monit. Comput. D 9 (December (4)) (1992) 257-267.

[183] J.R. Millan, J. Mourino, M. Franze, F. Concotti, M. Varsta, J. Heikkonen, F. Babiloni, A local neural classifier for the recognition of EEG patterns associated with mental tasks, IEEE Trans. Neural Netw. 13 (May (3)) (2002) 678-686.

[184] H. Cecotti, A. Graeser, Convolutional neural network with embedded Fourier transform for EEG classification, in: Proc. 19th Int. Conf. Patt. Recog. ICPR, 8-11 December, 2008, pp. 1-4.

[185] L. Larsen, E. Ruspini, J. McDew, D. Walter, D. Adey, A test of sleep staging system in unrestrained chimpanzee, Brain Res. 40 (1972) 319-343.

[186] J.C. Bezdek, Pattern Recognition with Fuzzy Objective Function Algorithms, Plenum, New York, 1981.

[187] I. Gath, E. Bar-On, Computerized method for scoring of polygraphis sleep recordings, Comput. Prog. Biomed. 11 (1980) 217-223.

[188] I. Gath, C. Feuerstein, A. Geva, Unsupervised classification and adaptive definition of sleep patterns, Pattern Recogn. Lett. 15 (October (10)) (1994) 977-984.

[189] G. Zouridakis, B.H. Jansen, N.N. Boutros, A fuzzy clustering approach to EP estimation, IEEE Trans. Biomed. Eng. 44 (August (8)) (1997) 673-680.

[190] X.L. Xie, G. Beni, A validity measure for fuzzy clustering, IEEE Trans. Pattern Anal. Mach. Intell. 13 (August (8)) (1991) 841-847.

[191] Z. Hilal Inan, M. Kuntalp, A study on fuzzy c-means clustering-based systems in automatic spike detection, Comput. Biol. Med. 37 (2007) 1160-1166.

[192] C. Xydeas, P. Angelov, S.-Y. Chiao, M. Reoullas, Advances in classification of EEG signals via evolving fuzzy classifiers and dependent multiple HMMs, Comput. Biol. Med. 36 (2006) 1064-1083.

[193] R. Harikumar, B.S. Narayanan, Fuzzy techniques for classification of epilepsy risk level from EEG signals, in: Proc.TENCON 2003, Conf. Conv. Tech. AsiaPacific Reg., vol. 1, 15-17 October, 2003, pp. 209-213.

[194] R. Allen, D. Smith, Neuro-fuzzy closed loop control of depth of anesthesia, Artif. Intell. Med. 21 (2001) 185-191.

[195] J.E. Heiss, C.M. Held, P.A. Estevez, C.A. Perez, C.A. Holzman, J.P. Perez, Classification of sleep stages in infants: a neuro fuzzy approach, IEEE Eng. Med. Biol. Mag. 21 (September-October (5)) (2002) 147-151.

[196] I. Guler, E.D. Ubeyli, Adaptive neuro-fuzzy inference system for classification of EEG signals using wavelet coefficients, J. Neurosci. Methods 148 (2005) $113-121$.

[197] S. Darvishi, A. Al-Ani, Brain-computer interface analysis using continuous wavelet transform and adaptive neuro-fuzzy classifier, in: Proc. 29th Int. Annu. Conf. IEEE Eng. Med. Biol. Soc., 22-26 August, 2007, pp. 3220-3223.

[198] A. Yildiz, M. Akin, M. Poyraz, G. Kirbas, Application of adaptive neuro-fuzzy inference system for vigilance level estimation by using wavelet-entropy feature extraction, Expert Syst. Appl. Int. J. 36 (May (4)) (2009) 7390-7399.

[199] G. Yang, Y. Lin, P. Bhattacharya, Multimodality inferring of human cognitive states based on integration of neuro-fuzzy network and information fusion techniques, EURASIP J. Adv. Sig. Proc. (2008), Art. ID 371621, Available from: http://www.hindawi.com/journals/asp/2008/371621.abs.html (open access).

[200] E.D. Ubeyli, Automatic detection of electroencephalographic changes using adaptive neuro-fuzzy inference system employing Lyapunov exponent, Expert Syst. Appl. Int. J. 36 (July (5)) (2009) 9031-9038

[201] N.Sadati, H.R. Mohseni, A. Maghsoudi, Epileptic seizure detection using neural fuzzy networks, IEEE Int. Conf. Fuzzy Syst. (2000) 596-600.

[202] H.-N.L. Teodorescu, A. Kandel, L.C. Jain, Fuzzy and Neuro-Fuzzy Systems in Medicine, CRC Press, Boca Raton, FL, 1999.

[203] G.A. Carpenter, S. Grossberg, N. Markuzon, J.H. Reynolds, D.B. Rosen, Fuzzy ARTMAP: a neural network architecture for incremental supervised learning of analog multidimensional maps, IEEE Trans. Neural Netw. 3 (September (5)) (1992) 698-713.

[204] R. Palaniappan, P. Raveendran, S. Nishida, N. Saiwaki, Evolutionary fuzzy ARTMAP for autoregressive model order classification of EEG signals, Proc. IEEE Int. Conf. Syst. Man Cybern. 5 (2000) 3682-3686. 
[205] R. Palaniappan, P. Raveendran, Cognitive task prediction using parametric spectral analysis of EEG signals, Malaysian J. Comput. Sci. 14 (1)(2001) 58-67.

[206] R. Palaniappan, R. Paramesran, S. Nishida, N. Saiwaki, A new brain-computer interface design using fuzzy ARTMAP, IEEE Neural Syst. Rehabil. Eng. 10 (September (3)) (2002) 140-148.

[207] R. Palaniappan, K.V.R. Ravi, Improved visual evoked potential classification for person recognition using PCA and normalization, Pattern Recogn. Lett. 27 (May (7)) (2006) 726-733.

[208] K.V.R. Ravi, R. Palaniappan, Neural network classification of late gamma band electroencephalogram features, Soft Comp. Fusion Found. Methodol. Appl. 10 (2) (2005) 163-169.

[209] D. Coyle, G. Prasad, T.M. McGinnity, Faster self-organizing fuzzy neural network training and a hyperparameter analysis for brain-computer interface, IEEE Trans. Syst. Man Cybern. Part B: Cybern. 39 (6) (2009) 1458-1471.

[210] Q. Xu, H. Zhou, Y. Wang, J. Huang, Fuzzy support vector machine for classification of EEG signal using wavelet-based features, Med. Eng. Phys. 31 (7) (2009) 858-865.

[211] R. Boostani, K. Sadatnezhad, M. Sabeti, An efficient classifier to diagnose of schizophrenia based on EEG signals, Expert Syst. Appl. 36 (April (3)) (2009) 6492-6499.

[212] B. Yang, G. Yan, T. Wu, R. Yan, Subject-based feature extraction using fuzzy wavelet packet in brain-computer interfaces, Signal Proc. 87 (2007) 1569-1574.

[213] V. Esmaeili, A. Assareh, M.B. Shamsollahi, M.H. Moradi, N.M. Arefian, Estimating the depth of anesthesia using fuzzy soft computation applied to EEG features, Intell. Data Anal. 12 (2008) 393-407.

[214] E. Huupponen, S.-L. Himanen, A. Varri, J. Hasan, A. Saastamoinen, M. Lehtokangas, J. Saarinen, Fuzzy detection of EEG alpha without amplitude thresholding Artif. Intell. Med. 24 (February (2)) (2002) 133-147.

[215] J. Petersen, G. Stockmanns, W. Nahm, EEG analysis for assessment of depth of anesthesia, in: P.S. Szczepaniak, P.J.G. Lisboa, J. Kacprzyk (Eds.), Fuzzy Systems in Medicine, Physica-Verlag, 2000, pp. 261-280.

[216] C.A. Pena-Reyes, M. Sipper, Evolutionary computation in medicine: an overview, Artif. Intell. Med. 19 (2000) 1-23.

[217] H.S. Lopes, Genetic programming for epileptic pattern recognition in electroencephalographic signals, Appl. Soft Comput. 7 (2007) 343-352.

[218] O. Smart, H. Firpi, G. Vachtsevanos, Genetic programming of conventional features to detect seizure precursors, Eng. Appl. Artif. Intel. 20 (December (8)) (2007) 1070-1085.

[219] C. Estebanez, J.M. Valls, R. Aler, I.M. Galvan, A first attempt at constructing genetic programming expressions for EEG classification, in: LNCS: Artificial Neural Networks: Biological Inspirations - ICANN 2005, Springer, Heidelberg 2005, pp. 665-670.

[220] R. Harikumar, R. Sukanesh, P. Arvindan Bharathi, Genetic algorithm optimization of fuzzy outputs for classification of epilepsy risk levels from EEC signals, in: Proc. TECON 2004 Conf. Conv. Tech. Asia-Pacific Reg., vol. 2, 21-24 November, 2004, pp. 1585-1589.

[221] R. Sukanesh, R. Harikumar, A comparison of genetic algorithm \& neural network (MLP) in patient specific classification of epilepsy risk levels from EEG signals, Eng. Lett. 14 (February (1)) (2007), Available from: http://www.engineeringletters.com/issues_v14/issue_1/EL_14_1_18.pdf.

[222] G.J. McLachlan, Discriminant Analysis and Statistical Pattern Recognition, John Wiley \& Sons, New York, 1992.

[223] F.H. Duffy, M.B. Denckla, P.H. Bartels, G. Sandini, L.S. Kiessling, Dyslexia: automated diagnosis by computerized classification of brain electrical activity, Ann. Neurol. 7 (5) (1980) 421-428.

[224] R.W. Thatcher, R.A. Walker, I. Gerson, F.H. Geisler, EEG discriminant analyses of mild head trauma, Electroencephalogr. Clin. Neurophysiol. 73 (1989) 94-106.

[225] R.W. Thatcher, D.M. North, R.T. Curtin, R.A. Walker, C.J. Biver, J.F. Gomez, A.M Salazar, An EEG severity index of traumatic brain injury, J. Neuropsychiatry Clin. Neurosci. February (13) (2001) 77-87.

[226] M.R. Nuwer, D.A. Hovda, L.M. Schrader, P.M. Vespa, Routine and quantitative EEG in mild traumatic brain injury, Clin. Neurophysiol. 116 (2005) 2001-2025.

[227] L.S. Prichep, E.R. John, QEEG profiles of psychiatric disorders, Brain Topogr. 4 (4) (1992) 249-257

228] A.D. Garson, L.C. Parra, P. Sajda, Cortical origins of response time variability during rapid discrimination of visual objects, Neurolmage 28 (November (2) (2005) 342-353.

[229] A.D. Garson, L.C. Parra, P. Sajda, Cortically coupled computer vision for rapid image search, IEEE Trans. Neural Syst. Rehabil. Eng. 14 (June (2)) (2006) 174-179.

[230] D.W. Hosmer Jr., S. Lemeshow, Applied Logistic Regression, John Wiley \& Sons, New York, 1989.

[231] B. Efron, The efficiency of logistic regression compared to normal discriminant analysis, J. Am. Stat. Assoc. 70 (December) (1975) 892-898.

[232] R.O. Duda, P.E. Hart, D.G. Stork, Pattern Classification, 2nd ed., John Wiley \& Sons, New York, 2001

[233] T. Hastie, R. Tibshirni, A. Buja, Flexible Discriminant Analysis by Optimal Scoring, AT\&T Bell Labs, Murray Hills, NJ, 1993, Available from: http://utstat.toronto.edu/reports/tibs/fda.ps.

[234] K. Majumdar, R.M. Frank, Three linear discriminators for separating scalp human EEG signals during RSVP tasks, unpublished work, 2006.

[235] V. Bostanov, BCI competition 2003 - data sets Ib and IIb: feature extraction from evenet related brain potentials with the continuous wavelet transform and the $t$-value scalogram, IEEE Trans. Biomed. Eng. 51 (June (6)) (2004) 1057-1061.

[236] S. Sun, C. Zhang, Y. Lu, The random electrode selection ensemble for EEG signal classification, Pattern Recogn. 41 (May (5)) (2008) 1663-1675.

[237] B. Blankertz, G. Curio, K. Muller, Classifying Single Trial EEG: Towards Brain Computer Interfacing. Advances in Neural Information Processing Systems (NIPS), MIT Press, Cambridge, MA, 2002, pp. 157-164.

[238] F. Lotte, M. Congedo, A. Lecuyer, F. Lamarche, B. Arnaldi, A review of classification algorithms for EEG-based brain-computer interface, J. Neural Eng. 4 (2) (2007) R1-R13.

[239] A. Alkan, E. Koklukaya, A. Subasi, Automatic seizure detection in EEG using logistic regression and artificial neural network, J. Neurosci. Methods 148 (October (2)) (2005) 167-176.

[240] A. Subasi, A. Alkan, E. Koklukaya, M.K. Kiymik, Wavelet neural network classification of EEG signals by using AR model with MLE preprocessing, Neural Netw. 18 (7) (2005) 985-997.

[241] R. Tomioka, K. Aihara, K.-R. Muller, Logistic regression for single trial EEC classification, in: B. Scholkopf, J. Platt, T. Hofmann (Eds.), Advances in Neura Information Processing Systems, vol. 19, MIT Press, Cambridge, MA, 2006, pp. 1402-1409.

[242] M. Gerez, A. Tello, Selected quantitative EEG (QEEG) and event-related potential (ERP) variables as discriminators for positive and negative schizophrenia Biol. Psychiatry 38 (1995) 34-49.

[243] J.C. Lind, Z.J. Koles, P. Flor-Henry, A.C.K. Soong, Frequency domain discriminant analysis of electroencephalogram, Psychophysiology 34 (May (3))(1997) 358-364.

[244] C.J.C. Burges, A tutorial on support vector machines for pattern recognition, Data Mining Knowl. Discov. 2 (1998) 121-167.

[245] J. Lofhede, N. Lofgren, M. Thordstein, A. Flisberg, I. Kjellmer, K. Lindecrantz, Classification of burst and suppression in the neonatal electroencephalogram, J. Neural Eng. 5 (2008) 402-410.

[246] L. Shoker, S. Sanei, J. Chambers, Artifact removal from electroencephalograms using a hybrid BSS-SVM algorithm, IEEE Signal Proc. Lett. 12 (October (10)) (2005) 721-724.

[247] M. Kaper, P. Meinicke, U. Grossekathoefer, T. Lingner, H. Ritter, BCI Competition 2003 - data set IIb: support vector machines for the P300 speller paradigm, IEEE Trans. Biomed. Eng. 51 (June (6)) (2004) 1073-1076.

[248] Y. Li, C. Guan, H. Li, Z. Chin, A self-training semi-supervised SVM algorithm and its application in an EEG-based brain computer interface speller system, Pattern Recogn. Lett. 29 (2008) 1285-1294.

[249] M. Thulasidas, C. Guan, J. Wu, Robust classification of EEG signal for braincomputer interface, IEEE Trans. Neural Syst. Rehabil. Eng. 14 (March (1)) (2006) 24-29.

[250] A. Schlogl, F. Lee, H. Bischof, G. Pfurtscheller, Characterization of four-class motor imagery EEG data for the BCI-competition 2005, J. Neural Eng. 2 (2005) L14-L22.

[251] W. Xu, C. Guan, C.E. Siong, S. Ranganatha, M. Thulasidas, J. Wu, High accuracy classification of EEG signal, in: Proc. 17th Int. Conf. Patt. Recog. ICPR, vol. 2 2004, pp. 391-394.

[252] S. Sun, C. Zhang, D. Zhang, An experimental evaluation of ensemble methods for EEG signal classification, Pattern Recogn. 28 (2007) 2157-2163.

[253] S. Baillet, L. Garnero, A Bayesian approach to introducing anatomo-functional priors in the EEG/MEG inverse problem, IEEE Trans. Biomed. Eng. 44 (May (5)) (1997) 374-385.

[254] G.S. Russell, R. Srinivasan, D.M. Tucker, Bayesian estimate of error bounds for EEG source imaging, IEEE Trans. Med. Imaging 17 (December (6)) (1998) 1084-1089.

[255] N.J. Trujillo-Barreto, E. Aubert-Vazquez, P.A. Valdes-Sosa, Bayesian model averaging in EEG/MEG imaging, Neurolmage 21 (2004) 1300-1319.

[256] D. Khosla, M. Don, M. Singh, Iterative Bayesian maximum entropy method for the EEG inverse, Proc. SPIE 3033 (1997) 147-158.

[257] J. Daunizeau, J. Mattout, D. Clonda, B. Goulard, H. Benali, J.-M. Lina, Bayesian spatio-temporal approach for EEG source reconstruction: conciliating ECD and distributed models, IEEE Trans. Biomed. Eng. 53 (March (3)) (2006) 503-516.

[258] G. Yildiz, A.D. Duru, A. Ademoglu, T. Demiralp, Bayesian EEG dipole source localization using SA-RJMCMC on realistic head model, in: Proc. 29th Annu. Int. Conf. IEEE Eng. Med. Biol. Soc., 22-26 August, 2007, pp. 4268-4277.

[259] K.J. Friston, W. Penny, C. Phillips, S. Kiebel, G. Hinton, J. Ashburner, Classical and Bayesian inference in neuroimaging: theory, Neurolmage 16 (2002) 465-483.

[260] K. Friston, L. Harrison, J. Daunizeau, S. Kiebel, C. Phillips, N. Trujillo-Barreto R. Henson, G. Flandin, J. Mattout, Multiple sparse priors for M/EEG inverse problem, Neurolmage 39 (2008) 1104-1120.

[261] K. Friston, R. Henson, C. Phillips, J. Mattout, Bayesian estimation of evoked and induced responses, Hum. Brain Map. 27 (2006) 722-735.

[262] J. Daunizeau, C. Grova, G. Marrelec, J. Mattout, S. Jbabdi, M. Pelegrini-Issac J.-M. Lina, H. Benali, Symmetrical event-related EEG/fMRI information fusion in a variational Bayesian framework, Neurolmage 36 (2007) 69-87.

[263] W.D. Penny, S.J. Roberts, Bayesian neural networks for detection of imagined finger movements from single-trial EEG, Tech. Report, Department of Electrical Engineering, Imperial College of London, 1997, Available from: www.robots.ox.ac.uk/ sjrob/Pubs/bayes-eeg.ps.gz.

[264] S.J. Roberts, W.D. Penny, Real-time brain computer interfacing: a preliminary study using Bayesian learning, Med. Biol. Eng. Comput. 38 (1) (2000) 56-61. 
[265] X. Zhu, C. Guan, J. Wu, Y. Cheng, Y. Wang, Bayesian method for continuous cursor control in EEG-based brain-computer interface, in: Proc. 27th Annu. Int. Conf. IEEE Eng. Med. Biol. Soc., Sanghai, 17-18 January, 2006, pp. 7052-7055.

[266] X. Zhu, J. Wu, Y. Cheng, Y. Wang, A unified framework to exploit information in BCI data for continuous prediction, Neurocomputing 71 (January (4-6)) (2008) 1022-1031.

[267] P. Shenoy, R.P.N. Rao, Dynamic Bayesian networks for brain-computer interfaces, in: Advances in Neural Information Processing Systems (NIPS), vol. 17 MIT Press, Cambridge, MA, 2005, pp. 353-360.

[268] S. Rezaei, K. Tavakolian, A.M. Nasrabadi, S.K. Setarehdan, Different classification techniques considering brain computer interface applications, J. Neural Eng. 3 (June (2)) (2006) 139-144.

[269] X. Lei, P. Yang, D. Yao, An empirical Bayesian framework for brain computer interface, IEEE Trans. Neural Syst. Rehabil. Eng. 17 (6) (2009) 521-529.

[270] S. Chiappa, 2006. Analysis and classification of EEG signals using probabilistic models for brain computer interfaces, PhD Thesis, Ecole Polytechnique Federale de Lausanne, Switzerland. Available from: http://silviachiappa.swisspowered.net/publications/silviachiappa06phd.pdf.

[271] Y. Bengio, P. Frasconi, Input-output HMM's for sequence processing, IEEE Trans. Neural Net. 7 (September (5)) (1996) 1231-1249.

[272] A. Flexer, P. Sykacek, I. Rezek, G. Dorffner, Using hidden Markov models to build an automatic, continuous and probabilistic sleep stager for the SIESTA project, in: Proc. IEEE Int. Joint Conf. Neural Net., 2000, pp. 3627-3631.

[273] G. Pfurtscheller, C. Neuper, Motor imagery and direct brain-computer communication, Proc. IEEE 89 (July (7)) (2001) 1123-1134.
[274] B. Obermaier, C. Guger, C. Neuper, G. Pfurtscheller, Hidden Markov models for online classification of single trial EEG data, Pattern Recogn. Lett. 22 (October (12)) (2001) 1299-1309.

[275] L.R. Rabiner, B.H. Juang, An introduction to hidden Markov models, IEEE Acoust. Speech Signal Proc. Mag. 3 (January (1)) (1986) 4-16, part 1.

[276] L.R. Rabiner, A tutorial on hidden Markov models and selected applications in speech recognition, Proc. IEEE 77 (February (2)) (1989) 257286.

[277] R.S. Huang, C.J. Kuo, L.-L. Tsai, O.T.C. Chen, EEG pattern recognition-arousal states detection and classification, in: Proc. IEEE Int. Conf. Neural Netw., vol. 2, 3-6 June, 1996, pp. 641-646.

[278] S. Zhong, J. Ghosh, HMM and coupled HMMs for multi-channel EEG classification, in: Proc. IJCNN'02, vol. 2, Honolulu, HI, USA, 2002, pp. 1154-1159, Available from: http://www.ideal.ece.utexas.edu/papers/zhong_ijcnn2002. pdf.

[279] H. Lee, S. Choi, PCA + HMM + SVM for EEG pattern classification, in: Proc.7th IEEE Int. Symp. Signal Proc. Appl., 1-4 July, 2003, pp. 541-544, Available from: http://www.postech.ac.kr/ seungjin/publications/isspa03.pdf.

[280] C. Zhang, C. Zheng, X. Yu, Y. Ouyang, Estimating VDT mental fatigue using multichannel linear descriptors and KPCA-HMM, EURASIP J. Adv. Sig. Proc. (2008), article ID 185638 (open access).

[281] S. Wong, A.B. Gardner, A.M. Krieger, B. Litt, A stochastic framework for evaluating seizure prediction algorithms using hidden Markov models, J. Neurophysiol. 97 (2007) 2525-2532. 